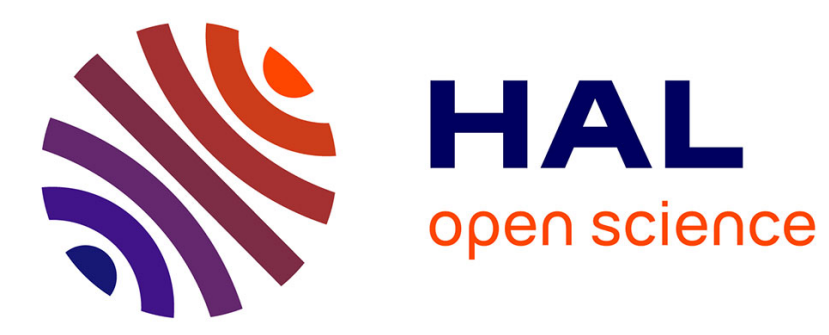

\title{
Représentation ego-centrée à base d'affordances de l'environnement pour la simulation multi-agents de trafic
}

Feirouz Ksontini, René Mandiau, Zahia Guessoum, Stéphane Espié

\section{To cite this version:}

Feirouz Ksontini, René Mandiau, Zahia Guessoum, Stéphane Espié. Représentation ego-centrée à base d'affordances de l'environnement pour la simulation multi-agents de trafic. RTS - Recherche Transports Sécurité, 2016, 2015 (03-04), pp.153-171. 10.4074/S0761898016004027 . hal-01670597

\section{HAL Id: hal-01670597 \\ https://hal.science/hal-01670597}

Submitted on 14 Mar 2018

HAL is a multi-disciplinary open access archive for the deposit and dissemination of scientific research documents, whether they are published or not. The documents may come from teaching and research institutions in France or abroad, or from public or private research centers.
L'archive ouverte pluridisciplinaire HAL, est destinée au dépôt et à la diffusion de documents scientifiques de niveau recherche, publiés ou non, émanant des établissements d'enseignement et de recherche français ou étrangers, des laboratoires publics ou privés. 


\title{
Représentation ego-centrée à base d'affordances de l'environnement pour la simulation multi-agents de trafic
}

\section{Affordance based ego-centered representation of environment for multi-agent traffic simulation}

\author{
Feirouz Ksontini - René Mandiau - Zahia Guessoum - Stéphane Espié \\ C IFSTTAR et Éditions NecPlus 2015
}

\begin{abstract}
Résumé Afin d'améliorer la validité des simulations de trafic en milieux urbain et péri- urbain, nous proposons un nouveau modèle d'agent permettant de mieux prendre en compte le contexte de conduite et les comportements des conducteurs en termes d'occupation de l'espace. Le but est de produire en simulation des comportements observés en situation réelle tels que les phénomènes de faufilement ( 2 roues, véhicules d'urgence). Nous dotons l'agent d'une représentation ego-centrée de l'environnement fondée sur le concept des affordances. Nous utilisons ainsi les affordances pour identifier les actions possibles, en termes d'occupation de l'espace (voies virtuelles), offertes par l'environnement. Nous les utilisons ensuite pour construire une représentation ego-centrée de la situation. Le modèle d'agent proposé a été implémenté avec ArchiSim. Cette implémentation a permis de reproduire une situation de trafic réelle et de comparer les données simulées avec celles recueillies sur le terrain.
\end{abstract}

Mots clés simulation multi-agents · affordances · simulation de trafic $\cdot$ voies virtuelles

\begin{abstract}
To improve the validity of traffic simulations in urban and suburban areas, we propose to consider the driving context and the driver behavior in terms of space occupation. We endow agent driver with an ego-centered
\end{abstract}

Feirouz Ksontini $(\bowtie)$

Ifsttar, TS2-SIMU 14-20 bd Newton, F-77447

e-mail : feirouz.ksontini@ifsttar.fr,

feirouz.ksontini@univ-valenciennes.fr

René Mandiau $(\square)$

Université de Valenciennes, LAMIH CNRS 8201, F-59313

Valenciennes, France

e-mail : rene.mandiau@univ-valenciennes.fr

Zahia Guessoum $(\triangle)$

LIP6, MAS Team, 4 place Jussieu, F-75005

e-mail : zahia.guessoum@lip6.fr

Stéphane Espié $(\square)$

Ifsttar, TS2-SIMU 14-20 bd Newton, F-77447

e-mail : stephane.espie@ifsttar.fr representation of the environment based on the concept of affordances. We use the affordances to identify the possible actions, in terms of space occupation (virtual lanes), afforded by the environment. We use them, next, to build an ego-centered representation of the situation. The proposed driver model was implemented with ArchiSim. The experiments allow to reproduce a real traffic situation and to compare simulated data to real ones.

Keywords Multi-agent simulation · affordances · traffic simulation · virtual lanes

\section{Introduction}

En matière de gestion de trafic routier, l'occupation de l'espace au sol est un problème de recherche bien connu. Dans les zones urbaines et dans un contexte de trafic dense, une mauvaise gestion de l'occupation de l'espace routier peut favoriser divers effets négatifs comme par exemple la congestion du trafic. La chaussée est souvent décomposée en voies de circulation et cette décomposition de l'espace routier est généralement respectée par les conducteurs. Cependant, la réalité montre que les pratiques des conducteurs humains ne sont pas toujours contraintes par cette gestion physique de l'espace routier. On peut aussi noter que de nombreux gouvernements européens étudient l'adaptation dynamique de l'espace routier et son influence sur le trafic. Ces études concernent par exemple la gestion dynamique des voies de circulation qui désigne les méthodes permettant d'augmenter ou de modifier le nombre de voies d'une infrastructure routière grâce à une redéfinition du marquage au sol en prenant en compte l'état du trafic. Un exemple consiste à changer dynamiquement le marquage au sol, en fonction de la densité du trafic et de sa vitesse, en utilisant des dispositifs lumineux.

Les Systèmes multi-agents (SMA) permettent la simulation de phénomènes complexes qui ne peuvent pas être facilement décrits analytiquement. Les SMA fournissent une solution aux problèmes posés par la simulation ou 
la gestion du trafic routier [1,2,3]. Cependant, pour la problématique de l'occupation de l'espace, les modèles existants considèrent souvent que le comportement d'un conducteur est normatif en termes d'occupation de l'espace. Ces modèles ne prennent pas en compte les phénomènes observés dans la réalité tels que les manœuvres de faufilement entre les véhicules ou l'allocation dynamique des voies. Nous nous intéressons à reproduire les pratiques observées en termes d'occupation de l'espace au sol en simulation.

Nous abordons, dans ce papier, la simulation de trafic en considérant une approche comportementale fondée sur les systèmes multi-agents. Cette approche est développée depuis une vingtaine d'années à l'Ifsttar (ex Inrets) dans le simulateur de trafic ArchiSim [4]. Nous proposons de doter les agents d'une représentation ego-centrée de l'environnement leur permettant de prendre des décisions en termes d'occupation de l'espace. Dans ce cadre, notre approche est fondée sur le concept des affordances - l'ensemble de toutes les possibilités d'action d'un environnement [5]. Nous utilisons une version étendue du concept des affordances en introduisant l'aspect cognitif dans le processus d'identification de ces dernières. Dans nos travaux, les affordances dépendent à la fois des propriétés des objets et/ou de l'environnement proposant les différentes possibilités d'action, mais aussi des caractéristiques individuelles des agents. La première partie de l'article présente un état de l'art succinct des travaux existants en simulation de trafic traitant de la problématique de l'occupation de l'espace. La deuxième partie expose ensuite le concept des affordances et la notion de voies virtuelles. La troisième partie décrit notre modèle de représentation ego-centrée de l'environnement fondé sur les affordances. Enfin, la dernière partie présente les expérimentations et les résultats obtenus.

\section{Occupation de l'espace en simulation de trafic}

Dans cette partie, nous présentons les travaux qui se sont intéressés à la problématique de l'occupation de l'espace au sol. Nous commençons par présenter un état de l'art des travaux qui ont traité cette problématique. Ensuite, nous détaillons les modèles développés dans le cadre d'ArchiSim à cet effet.

\subsection{Approches existantes pour étudier l'occupation de l'espace}

La question posée concerne la reproduction de pratiques observées en termes d'occupation de l'espace au sol disponible, en particulier pour des contextes de trafic très dense en milieu urbain ou face à des événements spécifiques (un véhicule mal garé, un véhicule d'urgence, etc.). On observe que les conducteurs ne respectent pas toujours la configuration donnée par les voies physiques définies par le marquage au sol et occupent l'espace disponible de façon à répondre à leurs buts tout en prenant en compte le contexte. Ce type de comportement est observé particulièrement chez les conducteurs des deux-roues motorisés $(2 \mathrm{RM})$ ou dans certaines situations, telles que la présence de véhicules d'urgence, pour les autres usagers. Afin d'améliorer la simulation de trafic hétérogène, nous avons besoin de comprendre le comportement des différents types de conducteurs [6].

En termes d'approches de simulation, nous pouvons distinguer deux familles : l'approche mathématique et l'approche comportementale [2]. L'approche mathématique consiste à mettre en équations des données relevées sur le terrain par le biais de capteurs (caméras, boucles magnétiques, etc.). L'approche comportementale ou « centrée individu » s'attache à modéliser et à reproduire le plus fidèlement possible les comportements et les interactions des différentes entités afin d'obtenir des phénomènes émergents de trafic réaliste. Des modèles utilisant des automates cellulaires ont également été introduits pour répondre à la problématique de simulation de trafic. À mi-chemin entre une approche mathématique et une approche comportementale, ces modèles consistent en une discrétisation de l'espace roulable sous forme d'une grille dont la taille des cellules peut varier suivant le niveau de détail désiré.

\subsubsection{Approche mathématique}

Il existe peu de travaux fondés sur l'approche mathématique traitant de la problématique de l'occupation de l'espace telle que nous pouvons l'observer dans la réalité. La majorité des travaux suppose que le positionnement des véhicules sur la chaussée est régi par les voies physiques définies par le marquage au sol et ce quelle que soit la situation étudiée $[7,8,9]$.

Quelques outils ont intégré le comportement des deuxroues motorisés dans leur simulation. Nous sous-entendons, un comportement différent des autres usagers de la route, en termes d'occupation de l'espace. VISSIM [10] intègre ainsi le comportement des deux-roues dans la modélisation en décrivant un mouvement latéral continu. Le conducteur choisit la position latérale qui lui assure le temps maximal à une collision avec un autre véhicule. Afin de trouver cette position, le conducteur décompose la largeur de la route en voies virtuelles. A notre sens, ces paramètres ne sont pas suffisants, étant donné que le choix de la position 
latérale cible est uniquement fondé sur une évaluation instantanée et qu'il n'intègre aucune anticipation de la situation routière. Dynataiwan [11] prend également en compte les deux-roues motorisés qui se déplacent selon des relations macroscopiques de flux. Ce modèle utilise une métrique donnant un ratio d'équivalence entre une voiture et les autres moyens de transport (PCE - Passenger Car Equivalent). Cette métrique mesure l'impact que peut avoir un mode de transport sur les variables de trafic telles que la densité, la vitesse, etc. Par exemple, les valeurs typiques de PCE sont de 1 pour une voiture et de 0,5 pour une moto. L'une des limites de cette approche consiste dans une définition uniforme de ce ratio d'équivalence alors qu'il devrait dépendre de la composition du trafic, de l'emplacement (intersection ou section courante), du degré de congestion, etc.

La modélisation mathématique du trafic routier est un domaine de recherche et développement à part entière et la littérature qui y est consacrée est extrêmement abondante. De ce fait de nombreux modèles mathématiques du trafic et de nombreux outils de simulation en ont été inspirés. L'approche mathématique et les outils qui en résultent sont limités quand il s'agit de modéliser un comportement individuel. D'une manière générale, cette approche prend difficilement en compte l'hétérogénéité du trafic routier et la problématique de l'occupation dynamique de l'espace roulable. Les lois obtenues sont liées aux caractéristiques physiques du tronçon sur lequel ont été effectuées les mesures (par exemple, longueur, nombre de voies, type de marquage au sol, réglementation).

\subsubsection{Approche par automates cellulaires}

Des modèles utilisant des automates cellulaires ont été introduits pour répondre à la problématique de l'occupation de l'espace, notamment pour le cas particulier des 2RM. Ces modèles ont été appliqués avec succès à la simulation de flux homogènes. Chaque cellule représente habituellement l'espace de base dont un véhicule a besoin. Afin de tenir compte de plusieurs types de véhicules dans un même modèle d'automate cellulaire, des tailles de cellules non identiques sont désignées pour représenter différents moyens de transport.

Ahuja [12] a utilisé un automate cellulaire pour simuler un flux de trafic hétérogène. Les écarts avant, arrière et latéraux acceptés par chaque type de véhicule ont été mesurés et ajoutés à la taille du véhicule pour représenter l'espace routier effectivement occupé par ce dernier. La taille d'une cellule a été définie en fonction de la largeur de l'espace routier nécessaire pour les véhicules. D'autres travaux $[13,14]$ ont utilisé les automates cellulaires pour décrire un trafic mixte contenant des deux-roues motorisés.
Afin de traiter des situations avec plus d'un moyen de transport, ces modèles ont utilisé des tailles de cellules non identiques pour représenter différents moyens de transport. Pour la simulation de trafic homogène, nous pouvons établir un ensemble de règles de comportement intuitivement compréhensibles pour simuler le déplacement des véhicules. Cet outil de simulation est, dans ce cas, capable de produire des résultats étonnamment réalistes [15]. Cependant, lorsque nous appliquons les automates cellulaires à un trafic hétérogène, il est difficile d'avoir des résultats aussi pertinents que dans le cas précédent. Dans ces modèles, la largeur des véhicules n'est pas considérée comme un facteur important et la distance latérale entre les véhicules n'est pas prise en compte. Une telle modélisation présente des limites dans la mesure où la position latérale et l'écart latéral sont des facteurs cruciaux pour les manœuvres de faufilement pour les deux-roues par exemple. En conclusion, les automates cellulaires présentent plusieurs avantages tels que la simplicité des règles permettant ainsi de traiter de grands systèmes. Cependant, Spicher et al. [16] montrent que leur expressivité est limitée et souvent mal adaptée à tous les types de situations, en particulier lorsque des entités indépendantes se déplacent dans un espace dynamique.

\subsubsection{Approche comportementale multi-agents}

Les modèles de simulation fondés sur l'approche comportementale s'appuient sur les travaux en psychologie de la conduite. Ces derniers analysent le comportement individuel des conducteurs et leurs pratiques et cherchent à mieux comprendre les motifs sous-jacents à leurs prises de décision [17]. L'hétérogénéité des comportements, pour les différents contextes étudiés, permet de faire émerger le comportement des flux de trafic et ce pour des situations pouvant être complexes [18]. Dans ce cadre, les modèles de simulation fondés sur les SMA offrent la possibilité de simuler les acteurs du système de trafic par des agents autonomes et leurs interactions, et ce de manière plus réaliste car prenant en compte les contextes des prises de décisions $[1,2,4,19]$.

Dans la simulation du trafic routier, en plus du déplacement longitudinal, nous prenons en compte le déplacement latéral qui s'effectue en général à travers les mécanismes de changement de voies [20,21,22,23]. Dans ces travaux, les voies considérées correspondent aux voies physiques définies par le marquage au sol. Les conducteurs occupent l'espace en se référant uniquement à ces voies et se positionnent toujours au milieu de leurs voies. Dans ce cadre, la représentation que se fait le conducteur de son environnement correspond à cette configuration physique et elle est la même pour tous les usagers. Cette modélisation de l'environnement ne permet 
pas de reproduire les phénomènes d'occupation de l'espace et produit de ce fait des simulations qui ne correspondent pas forcément à ce que l'on peut observer dans la réalité.

Néanmoins, il $\mathrm{y} \mathrm{a}$ eu très peu de travaux qui se sont intéressés à la problématique de l'occupation de l'espace telle que nous pouvons l'observer dans certaines situations ou pour un certain type d'usagers. Bonte et al. [24] et Lee et al. [25] ont proposé des modélisations pour le cas particulier des deux-roues motorisés et ont essayé d'intégrer les spécificités de ces derniers en termes de déplacement et de positionnement sur la chaussée. Les travaux de Bonte et al. [24] ont été développé dans le cadre de la simulation de trafic ArchiSim et seront détaillés dans la section 2.3.2. Lee et al. [25] s'appuient sur une modélisation mathématique des règles d'interaction. Ces derniers ont considéré le modèle de poursuite de Gipps [26] pour décrire aussi bien le déplacement des voitures que celui des deux-roues motorisés ( $2 \mathrm{RM}$ ) avec quelques ajustements pour prendre en compte les paramètres cinématiques des 2RM et permettre un déplacement latéral plus adéquat. Ces modèles ont par la suite été utilisés dans un modèle de simulation à base d'agents. Les auteurs évoquent le concept des voies virtuelles sans les modéliser explicitement. La modélisation proposée par Lee et al. [25] reste tout de même une solution non générique développée uniquement pour un type particulier d'usager et par conséquent ne peut pas s'appliquer dans d'autres situations notamment des situations de blocage liées à un événement particulier, pour les gares de péages, etc. De plus, leur modèle est fondé sur le modèle de déplacement de Gipps [26] qui correspond à une modélisation mathématique du comportement et qui ne permet pas de prendre en compte un nombre élevé de paramètres des conducteurs et permettre ainsi une modélisation fine du comportement individuel des agents.

\subsection{Un problème de représentation de l'environnement}

Afin de proposer une solution générique qui prend en compte les pratiques des conducteurs en termes d'occupation de l'espace, nous nous sommes intéressés à la manière avec laquelle un conducteur se représente l'environnement autour de lui avant de prendre sa décision. En termes de représentation de l'environnement, les travaux existants sont fondés sur deux approches : les représentations allo-centrées et ego-centrées [27,28].

Dans les représentations ego-centrées, les relations spatiales sont généralement directement liées à l'agent qui se construit sa représentation en utilisant un système de référence avec des termes tels que, par exemple, à gauche, à droite, en face, ou derrière. Quand le contexte change, toutes les relations spatiales doivent être mises à jour. Alors que dans une représentation allo-centrée les entités de l'environnement sont localisées indépendamment de la position courante de celui qui se construit la représentation. Elle permet de définir la relation directe entre chacun des objets de l'espace. Elle est de nature flexible, puisque la localisation des objets ne change pas lorsque le sujet se déplace dans l'environnement. Les représentations allocentrées sont plus stables, mais sont plus difficiles à acquérir. De plus, le nombre des relations spatiales considérées est beaucoup plus élevé puisqu'il faut prendre en compte toutes les relations entre les différents objets de l'environnement. Dans une représentation ego-centrée, le nombre de ces relations peut être nettement moins important [28].

Dans la réalité, le conducteur « découvre » les situations au fur et à mesure qu'il se déplace. Il a donc besoin de percevoir son environnement pour prendre une décision. Il a ainsi tendance à se représenter les autres conducteurs par rapport à sa position (devant moi, à ma gauche, à ma droite, la distance qui me sépare de celui qui est juste devant, etc.). De ce point de vue, une représentation ego-centrée est donc plus intuitive dans le contexte de la simulation de trafic, pour laquelle nous avons besoin d'avoir une représentation contextuelle et dynamique de son propre environnement. De plus, la représentation ego-centrée est adaptée aux contextes dynamiques car le nombre de relations à mettre à jour est moins élevé que dans le cas de la représentation allo-centrée.

El Hadouaj et al. [29] ont utilisé une représentation ego-centrée de l'environnement dans le cadre de l'outil de simulation de trafic ArchiSim. Ces travaux sont détaillés dans la section suivante.

\subsection{Représentation de l'environnement dans ArchiSim}

Le projet ArchiSim [4], initié dès 1992, consiste à considérer le trafic routier comme un phénomène émergent résultant des actions et des interactions entre les différents acteurs de la route : les usagers (automobilistes, motards, piétons, etc.), l'infrastructure routière et la signalisation. Chaque acteur du système de trafic est un agent disposant d'objectifs et de caractéristiques propres, et doté d'autonomie lors de son déplacement sur le réseau routier simulé. La prise de décision de chaque agent est individuelle et se fonde sur une perception locale de son environnement.

\subsubsection{Représentation ego-centrée de l'environnement}

L'approche comportementale développée dans ArchiSim s'appuie sur les travaux en psychologie de la conduite effectués par Saad [17]. D'après ces études, l'espace autour du véhicule, constituant le champ de contrôle du conducteur, peut être décomposé en plusieurs secteurs, distingués selon leur localisation (avant, arrière, latérale gauche, latérale 
droite) et leur proximité (très proche, proche, lointain, très lointain). Dans le modèle proposé par Saad et Schnetzler [30], seules les zones situées sur la voie courante du véhicule, celles situées sur la voie immédiatement à droite et sur la voie immédiatement à gauche sont utilisées.

Par la suite, El Hadouaj [21] a extrapolé ce même raisonnement et a ajouté des zones de contrôle qui couvrent les voies non immédiatement adjacentes, à droite et à gauche, afin de prendre en compte l'état du trafic sur ces voies et permettre la levée de situations de blocage pour des autoroutes à plus de trois voies de circulation (figure 1). Elle considère ainsi au maximum 5 voies : la voie courante de l'agent, deux voies adjacentes à gauche et deux voies adjacentes à droite. Ces voies sont des voies physiques définies par le marquage au sol. Elle ne permet donc pas d'identifier les espaces libres sur la chaussée (autre que les espaces vides sur les voies physiques) ni de reproduire les pratiques observées en termes d'occupation de l'espace dans la simulation comme, par exemple, le comportement des deux-roues motorisés.

L'idée d'El Hadouaj [21] de faire une présélection sur l'ensemble des voies existantes pour en garder les plus pertinentes est intéressante dans la mesure où l'ensemble des solutions possibles est limité. Mais le choix des voies à retenir ne peut pas permettre de débloquer n'importe quelle situation notamment des situations de blocage liées à des accidents.

\subsubsection{Concept des voies virtuelles}

Par la suite, Bonte et al. [24] ont proposé une modélisation pour le cas particulier des 2RM afin de reproduire leurs comportements spécifiques tels que les faufilements entre les véhicules ou les remontées de files. Pour représenter les espaces libres sur la chaussée, les auteurs introduisent le concept des voies virtuelles. Elles sont désignées par « virtuelles» car elles n'existent pas physiquement sur la route, elles sont propres à un individu dans une situation et elles émergent selon le contexte. Les auteurs proposent un algorithme pour le découpage de la route en bandes libres et bandes occupées. Deux types de voies virtuelles ont ainsi été définis : des voies virtuelles interdites et des voies virtuelles autorisées. Une voie interdite est une voie virtuelle qu'un deux-roues doit éviter d'emprunter. Ces voies sont définies grâce à la présence des véhicules. Au contraire, une voie autorisée est une voie virtuelle qu'un deux-roues peut emprunter. Ces voies sont définies grâce à l'espace libre sur la route.

Dans le modèle de Bonte et al. [24], seul le paramètre de la taille du véhicule est pris en compte dans l'identification des voies virtuelles. L'évaluation des voies ne prend pas en compte par exemple un paramètre sur la distance par rapport à la réglementation du conducteur. Ce type de paramètre peut avoir un impact sur notre prise de décision, particulièrement dans le cas de pratiques non normatives. $\mathrm{Au}$ niveau de la représentation que se construit l'agent de son environnement, les auteurs font une décomposition systématique et géométrique en voies libres et occupées de tout l'espace routier en fonction de la taille des véhicules et de leur position sur la chaussée. D'une part, cette décomposition amène à un nombre variable et parfois très élevé de voies virtuelles, plus particulièrement dans le cas de routes à plusieurs voies. D'autre part, les auteurs utilisent les zones de contrôle définies initialement par El Hadouaj [21].

Plus généralement, une grande partie des outils de simulation existants ne supposent que le positionnement des véhicules sur la chaussée est régi par les voies physiques. Cette modélisation ne permet pas de simuler les phénomènes observés en termes d'occupation de l'espace roulable. Très peu de travaux en simulation multi-agents de trafic se sont intéressés à ces pratiques. Ils ont essentiellement modélisé le comportement des deux-roues motorisés pour qui ces pratiques sont fréquentes. Dans ce cadre, nous avons présenté essentiellement les travaux de Bonte et al. [24] et de Lee et al. [25]. Les deux solutions ont été développées pour le cas particulier des deux-roues motorisés et peuvent difficilement s'appliquer à d'autres types de véhicule. Pour remédier à ces limites, nous nous sommes intéressés à la manière avec laquelle un conducteur se représente l'environnement autour de lui.

Les deux sections suivantes sont consacrées à la proposition d'un modèle d'agent permettant de rendre compte des comportements d'occupation de l'espace que nous pouvons observer dans la réalité en se fondant sur une représentation ego-centrée de l'environnement de l'agent.

\section{Une vue générale du modèle d'agent à base d'affordances}

Afin de construire sa représentation ego-centrée de l'environnement, l'agent identifie les actions possibles offertes par l'environnement en termes d'occupation de l'espace. Nous proposons d'utiliser le concept des affordances afin de déterminer ces possibilités. Ainsi, notre représentation ego-centrée de l'environnement est composée par les affordances identifiées. Par exemple, si un conducteur n'a pas la possibilité d'aller tout droit sur sa voie (par exemple, dû à une fermeture de la voie), il ne va pas nécessairement ajouter cette voie à sa représentation mentale de l'environnement étant donné que cette voie est devenue interdite. Nous pouvons aussi considérer le comportement de faufilement des deux-roues motorisés : s'il y a suffisamment d'espace entre deux 


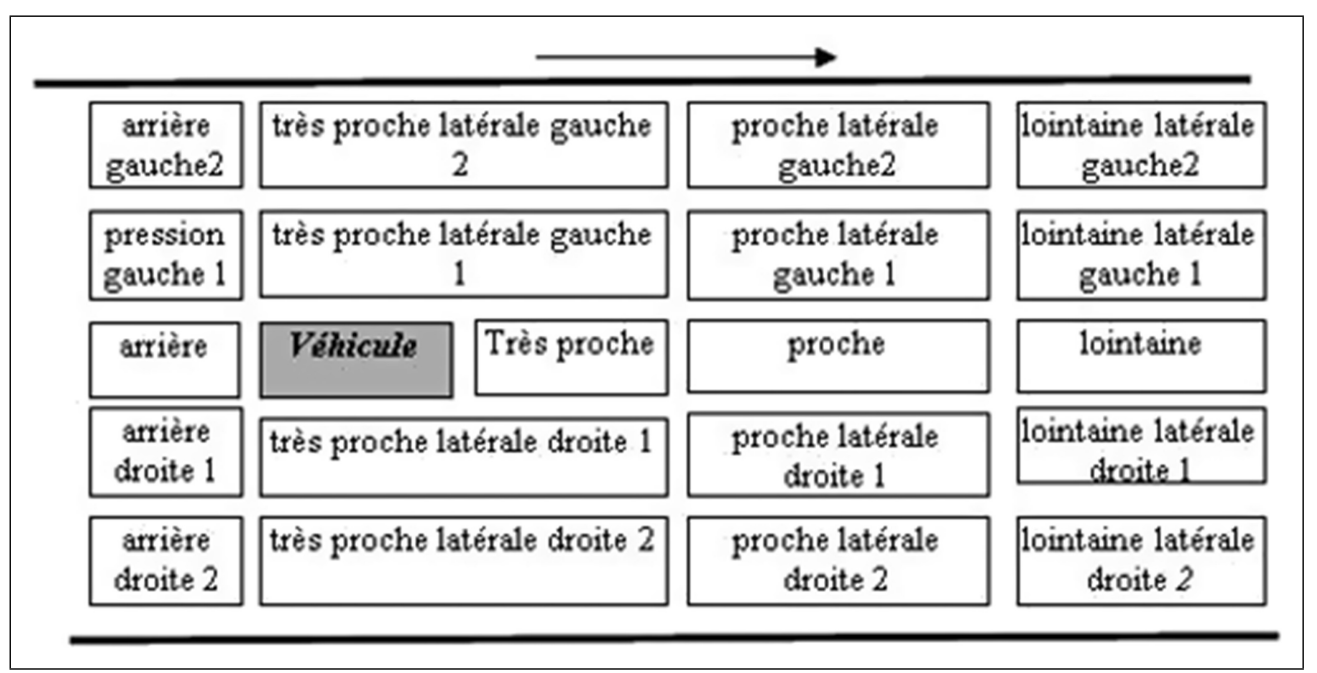

Fig. 1 Zones de contrôles d'un conducteur simulé d'après El Hadouaj [21]

files de voitures, la voie émergente devrait compléter la représentation ego-centrée que se construit le conducteur de son environnement, étant donné qu'elle lui offre une possibilité d'action différente de celle fournie par les voies physiques définies par le marquage au sol.

\subsection{Les affordances}

Les affordances sont issues de la théorie de la perception, développée initialement par le psychologue Gibson [5] qui considère que l'environnement offre à l'agent en mouvement des significations directement utiles pour l'action, des « disponibilités physiques perceptibles », qu'il appelle les affordances. Ce dernier les décrit comme étant des possibilités d'actions ou des opportunités que les agents peuvent percevoir dans l'environnement. Cette description suggère fortement un ensemble d'exigences de conception spécifiques pour gérer les interactions entre l'agent et son environnement. Cela ne comprend pas seulement l'exigence de conception que les percepts et les actions des agents soient à la fois explicitement représentés mais englobe également la nécessité pour les agents de percevoir les possibilités d'action directement accessibles dans l'environnement.

Duchon et al. [31], Norman [32] et Raubal [33] ont souligné que la théorie de Gibson est insuffisante pour expliquer la perception parce qu'elle néglige les processus de cognition. Duchon et al. [31] ont ainsi explicitement soutenu que l'agent ne se contente pas de répondre à un stimulus perçu directement par l'action qui est prévue dans cette situation. Il peut plutôt utiliser les informations fournies par les affordances d'une situation et raisonner sur elles d'une manière orientée vers les buts en choisissant les actions offertes qui mèneront à son objectif. D'autres auteurs considèrent que les affordances sont les résultats de l'interprétation mentale des choses, fondée sur la connaissance des agents. Norman [32] a aussi repris et étendu le concept de Gibson dans le but de concevoir des systèmes homme-machine. Ce dernier décrit les affordances perçues comme l'ensemble des actions qu'une entité a conscience de pouvoir faire. Enfin, Raubal [33] utilise une théorie étendue des affordances dans un modèle d'agent fonctionnel. L'auteur complète la théorie de la perception de Gibson avec des éléments de la cognition, des aspects situationnels et des contraintes sociales. Compte tenu de ces différents points de vue sur les affordances, nous soutenons l'idée de la prise en compte de la cognition pour modéliser les affordances. Nous ne pensons pas que la perception des affordances correspond uniquement à une perception directe, cela n'exclut pas pour autant qu'il puisse y avoir la nécessité d'un traitement perceptif.

Le concept des affordances a été utilisé dans plusieurs travaux, notamment dans le domaine de la robotique [34,35], dans les systèmes multi-agents pour des problématiques liées à la navigation de piétons [36], pour la simulation d'opérations militaires [37] ou pour la représentation de l'action située dans les systèmes agricoles [38].

Dans l'approche de Papasimeon et al. [37], le concept des affordances est intégré à une architecture $\mathrm{BDI}^{1}$. Afin d'introduire ce concept dans une telle architecture, les auteurs considèrent que les affordances sont traitées de la même manière que les attitudes mentales classiques telles que les croyances, les désirs et les intentions. Les affordances associent à un objet de l'environnement une intention possible que l'agent peut exécuter sous certaines conditions. L'avantage de ce type d'approche est qu'il

\footnotetext{
${ }^{1}$ Belief-Desire-Intention
} 
semble relativement facile de modéliser formellement le concept des affordances comme une attitude mentale dans des modèles d'agent existants. Toutefois, ce concept reste difficile à manipuler en simulation.

Dans une problématique qui se rapproche de la nôtre, Kapadia et al. [36] proposent un modèle de navigation et planification de trajet pour la simulation de piétons. Dans le cadre de ces travaux, les affordances correspondent aux différentes possibilités d'interaction entre un agent et son environnement. Chaque agent perçoit son environnement à travers un ensemble d'attributs vectoriels et scalaires avec un modèle de représentation ego-centrée. Les auteurs calculent ensuite des affordances en fonction des zones de perception identifiées. Un attribut d'affordances quantifie la valeur pouvant être attribuée à une affordance (une action) en prenant en compte les buts de l'agent. L'action ayant la valeur optimale correspond à la décision qui sera prise par l'agent. Dans cet article, les auteurs représentent les informations de l'environnement sous forme de champs de perception ego-centrés. Dans cette représentation, les zones prises en compte correspondent uniquement à l'environnement proche de l'agent. Cette proposition ne permet pas de prendre en compte tout le champ de perception qui pourrait avoir un impact sur la prise de décision de l'agent : il n'est donc pas possible d'anticiper des événements quelconques lors de la prise de décision.

Le concept d'affordances n'a pas été utilisé, à notre connaissance, pour la simulation du trafic routier. L'utilisation de ce concept dans le cadre de la simulation permet de mieux identifier les interactions entre les agents et les entités. Nous pensons que ce concept s'adapte bien à la problématique de la simulation de trafic routier, notamment pour l'occupation de l'espace. Les affordances identifient les actions possibles offertes par l'interaction des entités perçues et l'environnement dans lequel elles évoluent en termes d'occupation de l'espace.

Si nous nous référons à la définition des affordances, donnée par Gibson [5], ces dernières doivent être définies dans l'environnement et non pas dans la mémoire procédurale des agents. Pourtant un agent doit pouvoir raisonner sur les affordances pour prendre sa décision. Dans ce sens, Papasimeon [37] propose deux approches différentes pour modéliser les affordances. La première s'appuie intégralement sur le concept des affordances présenté par Gibson [5] et stocke toutes les affordances dans l'environnement ; la deuxième considère une définition plus souple et considère que les affordances sont dans la mémoire de l'agent.

La première approche a un certain nombre d'avantages. Le plus important est qu'elle correspond à une représentation plus proche du concept des affordances comme décrit en psychologie écologique. Un autre avantage consiste dans le fait que cette proposition accorde une place plus importante à l'environnement permettant ainsi de transférer une partie de l'intelligence au niveau de l'environnement. Cependant, dans le cadre de cette proposition, le modèle de l'environnement nécessite de pouvoir saisir toutes les relations nécessaires au calcul des affordances (incluant les informations concernant les agents individuels) ainsi que de déterminer les affordances pour chaque agent. Les agents de leur côté doivent être capables de percevoir les affordances, de les évaluer et décider laquelle adopter. De plus, l'architecture de simulation doit être en mesure de fournir les mécanismes d'interfaçage appropriés entre l'agent et l'environnement pour permettre à l'agent de percevoir les affordances pertinentes dans l'environnement. Le principal inconvénient de cette approche est que l'impact de la conception se traduit par un grand nombre de sous-systèmes au niveau de l'architecture de simulation. Par conséquent, l'introduction des affordances ne signifie pas seulement une reconsidération de la conception de l'agent et de l'environnement mais aussi l'interaction entre les deux. Ceci peut s'avérer difficile dans le cas où les affordances sont introduites dans une simulation déjà existante nécessitant ainsi de multiples modifications tant au niveau de l'agent qu'au niveau de l'environnement.

L'avantage de la deuxième modélisation des affordances réside dans le fait qu'il est plus facile d'introduire le concept des affordances dans une simulation multi-agents existante, les affordances peuvent ainsi être introduites au niveau du processus de raisonnement de l'agent sans affecter le reste de l'architecture.

Un autre aspect important à prendre en compte concerne le nombre d'affordances à calculer. Dans le cas le plus général, les affordances de chaque agent sont considérées par rapport à chaque entité présente dans l'environnement. Cette approche a l'avantage d'être générale. Toutefois, avec un grand nombre d'agents et d'entités, ce type d'approche est problématique du point de vue de la lourdeur du calcul en raison du grand nombre des relations possibles à examiner et à évaluer. Ce qui relève de la résolution de problèmes combinatoires. En réduisant le nombre d'affordances à examiner et à évaluer, il est possible d'améliorer de manière significative les performances de calcul de la simulation.

D'une manière générale, l'état de l'art nous a permis d'identifier les avantages et les inconvénients de chaque approche. Dans le cadre de notre application, nous optons pour la solution consistant à considérer que les affordances sont contenues dans la mémoire de l'agent dans la mesure où l'agent a besoin de raisonner sur les affordances perçues afin d'identifier celles qui peuvent correspondre réellement à des possibilités d'action dans l'environnement.

Nous proposons, ainsi, un modèle d'agent pour la problématique de l'occupation de l'espace fondé sur le concept des affordances. Les affordances identifiées permettent à l'agent de se construire une représentation 
ego-centrée de l'environnement qui prend en compte les possibilités d'occupation de l'espace facilitant ainsi la prise de décision de l'agent. Le fait de considérer les affordances du point de vue de l'agent (comme des constructions mentales internes) rend plus facile la mise en œuvre et l'implémentation de ce concept dans une simulation multi-agents que dans le cas où les affordances sont représentées comme un concept directement perceptible dans l'environnement.

Dans notre modélisation des affordances, nous utilisons une version étendue de ce concept. Cette version a été enrichie par la prise en compte de la cognition. Ainsi, les affordances, dépendent des contraintes de l'environnement en plus des capacités mentales de l'agent. Nous prenons en compte l'interprétation situationnelle de l'environnement par l'agent. Afin d'identifier les opportunités offertes par l'interaction des entités perçues avec l'environnement, l'agent prend en compte le contexte, les propriétés des autres agents et ses propres caractéristiques (capacités, intentions, buts, caractéristiques comportementales telles que la distance à la norme). Dans le cadre de notre application, nous supposons que les affordances correspondent aux voies possibles qui émergent à partir de l'environnement routier.

\subsection{Les voies virtuelles}

Le fait que l'infrastructure soit structurée «physiquement » par le biais du marquage au sol, n'empêche pas les conducteurs de surcharger la structure proposée en redéfinissant l'espace en fonction de leurs buts et du contexte routier. Nous considérons que chaque agent peut «plaquer » sur l'infrastructure existante une structure qui répond à ses besoins et qui correspond, de ce fait, à sa propre interprétation de la situation. De ce point de vue, nous réutilisons le concept des «voies virtuelles », proposé notamment par Bonte et al. [24].

Nous proposons d'adopter le concept des voies virtuelles pour structurer l'environnement [39]. Ces voies virtuelles correspondent, selon nous, aux affordances qui s'offrent à l'agent en termes de possibilités d'occupation de l'espace au sol. Les affordances identifiées serviront à construire une représentation ego-centrée dynamique et contextuelle de l'environnement fondée sur les différentes possibilités offertes par l'interaction entre les différents usagers de la route et l'environnement. Les agents n'interprètent pas les possibilités offertes par l'environnement de la même façon. De ce fait, les représentations d'une même situation routière peuvent différer selon le point de vue de l'agent. Par exemple, pour une même situation, un automobiliste et un motocycliste ne vont pas interpréter de la même manière les espaces libres qui peuvent émerger sur la chaussée. Cette différence est essentiellement dûe non seulement aux caractéristiques physiques des véhicules (taille, mobilité) mais aussi aux comportements individuels (degré de violation de la norme de chaque individu).

Chaque voie virtuelle est désignée par le terme $V V_{\mathrm{i}}$, où i désigne l'indice de la voie, et est caractérisée par un ensemble de propriétés $P_{\mathrm{i}}=\left\{p_{1 \mathrm{i}}, p_{2 \mathrm{i}}, \ldots, p_{\mathrm{qi}}\right\}$. Ces propriétés peuvent être décomposées en trois classes : des propriétés physiques, des propriétés liées aux flux et des propriétés de l'environnement adjacent à gauche et à droite.

Les propriétés physiques d'une voie correspondent aux propriétés permettant de délimiter l'espace physique occupé par cette voie. Elles sont données par la position de début, la position de fin, la largeur et la longueur de la voie. La longueur d'une voie est donnée par la distance à l'obstacle, c'est-à-dire, la distance à laquelle elle reste accessible (avant de rencontrer un obstacle tel qu'un autre véhicule ou avant la fermeture de la voie). La figure 2 illustre ces propriétés.

Dans cet exemple, les voies virtuelles sont déterminées par l'agent dans un contexte de 4 agents. Prenons l'exemple de la voie virtuelle sa largeur est donnée par l'espace disponible entre les deux véhicules formant cette voie et sa longueur est donnée par la distance séparant l'agent qui détermine les voies virtuelles à partir de jusqu'à l'obstacle.

Les propriétés liées au flux correspondent à une caractérisation du trafic parcourant la voie. Ces propriétés sont données par : la densité du trafic donnée par le nombre de véhicules circulant sur la voie en question, la vitesse moyenne pratiquée par les véhicules et l'écart type de la vitesse pratiquée mesurant la distribution de vitesses des véhicules qui roulent sur la voie et par conséquent la stabilité du trafic en termes de vitesse. Un écart type élevé supposerait que le trafic est instable, donc non prévisible.

En plus des caractéristiques physiques et celles liées au flux, nous avons aussi retenu d'autres propriétés, qui à notre sens sont importantes pour caractériser une voie d'autant plus qu'elles ont un impact sur le comportement de l'agent. Ces propriétés sont liées à «l'effet paroi ». Des travaux en psychologie de la conduite mettent en évidence l'impact de cet effet sur la vitesse pratiquée par le conducteur ainsi que son positionnement latéral sur les voies $[40,41]$.

L'effet paroi représente donc l'impact de l'environnement adjacent (voies adjacentes ou le bord de la route) sur le comportement du conducteur. Il peut être lié aux caractéristiques de l'infrastructure (largeur des voies, les murs d'un tunnel, etc.) ou au contexte routier (l'effet de l'existence de poids lourds sur les voies adjacentes, la variabilité des vitesses sur les voies adjacentes, etc.). Nous retenons les caractéristiques suivantes : la vitesse de chaque paroi (c'est-à-dire la vitesse du véhicule le plus lent de la 


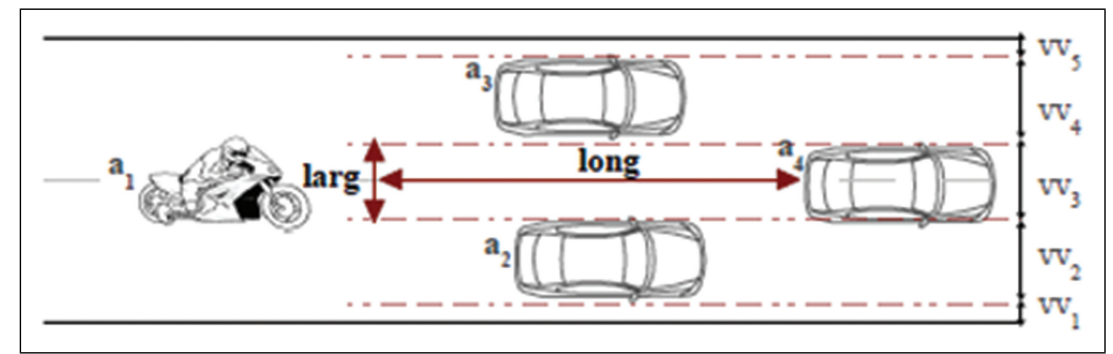

Fig. 2 Illustration des propriétés d'une voie

file qui définit la paroi ou une vitesse nulle s'il s'agit d'un bord de route), la stabilité des parois (c'est-à-dire, donnée par la différence entre les vitesses moyennes de chaque paroi et nous postulons que plus les vitesses des parois droite et gauche sont identiques et plus l'effet paroi peut être considéré comme stable) et la proximité des parois (c'est-à-dire, l'espace disponible entre le véhicule considéré et les bords de la voie). Ces caractéristiques agissent sur la position latérale et la vitesse appliquée.

\section{Un modèle d'agent fondé sur les affordances}

Nous proposons un modèle d'agent permettant de rendre compte des comportements d'occupation de l'espace tels que nous pouvons les observer dans la réalité. Notre modèle repose sur une représentation ego-centrée de l'environnement fondée sur les affordances [42]. Ces dernières permettent d'identifier les actions possibles offertes par l'environnement en termes d'occupation de l'espace. Dans le cadre de notre application, les agents suivent des voies pour se déplacer. Nous avons conservé cette notion de voie de déplacement dans notre modélisation de l'environnement et nous supposons que les affordances correspondent aux voies émergentes des interactions entre les usagers et l'environnement. Ces affordances dépendent à la fois du contexte de la situation routière et des caractéristiques individuelles de chaque agent. Les possibilités offertes par l'environnement ne sont pas interprétées de la même manière par tous les agents. Les affordances identifiées permettent à l'agent de se construire une représentation ego-centrée de l'environnement orientée vers les actions possibles.

Notre proposition des affordances a été enrichie par la prise en compte de la cognition. Ainsi, les affordances, dépendent des contraintes de l'environnement en plus des capacités mentales de l'agent. Nous prenons en compte l'interprétation situationnelle de l'environnement par l'agent. Afin d'identifier les opportunités offertes par l'interaction des entités perçues avec l'environnement, l'agent prend en compte le contexte, les propriétés des autres agents et ses propres caractéristiques (capacités,

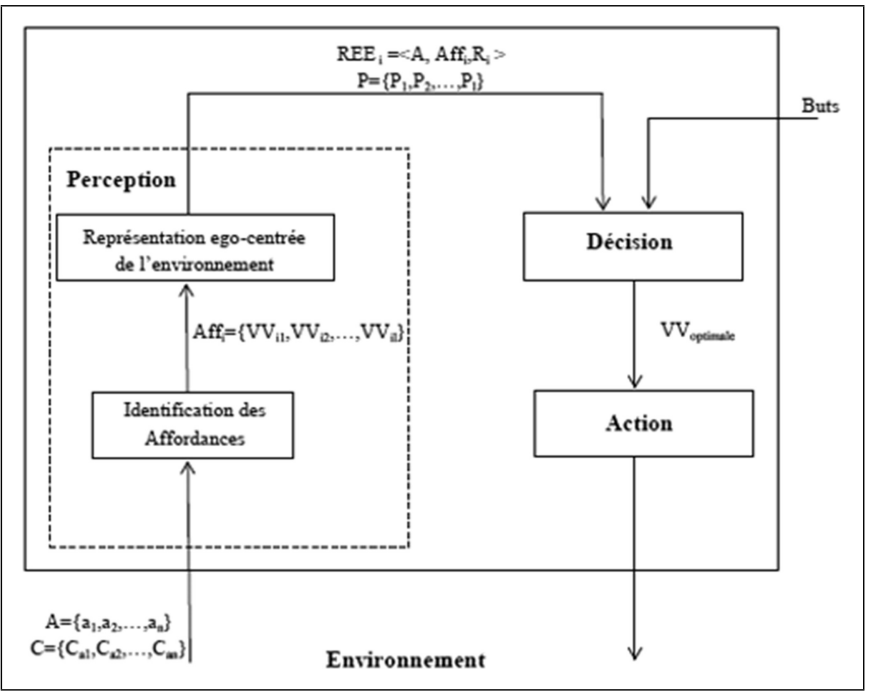

Fig. 3 Architecture de l'agent

intentions, buts, caractéristiques comportementales telles que la distance à la norme ${ }^{2}$ ).

Le modèle de l'agent est décomposé en trois modules se référant à l'architecture « Perception-Décision-Action » du modèle de prise de décision standard (figure 3).

\subsection{La perception}

L'agent perçoit les autres agents situés dans l'environnement, autour de lui, à une certaine distance de vision qui dépend de sa vitesse. Soit $A=\left\{a_{1}, a_{2}, \ldots\right.$, $\left.a_{\mathrm{n}}\right\}$ le sous-ensemble des agents perçus par $a_{\mathrm{i}}$. L'agent peut aussi percevoir quelques caractéristiques des autres agents telles que la position, le type, la vitesse de déplacement, etc. notées par l'ensemble $C=\left\{C_{1}, C_{2}, \ldots, C_{\mathrm{n}}\right\}$. Chaque $C_{\mathrm{i}}=\left\{C_{1 i}, C_{2 i}, \ldots, C_{\mathrm{m} i}\right\}$ est l'ensemble des propriétés décrivant chaque agent $a_{1} \in A$.

\footnotetext{
${ }^{2}$ La distance à la norme est spécifiée d'une manière aléatoire lors de l'initialisation de l'agent, elle donne le degré du respect de la norme et assure ainsi une hétérogénéité entre les agents.
} 
Soit $A f f_{\mathrm{i}}=\left\{V V_{1}, V V_{2}, \ldots, V V_{1}\right\}$ l'ensemble des affordances identifiées par l'agent $a_{\mathrm{i}}$. La détermination des affordances nécessite un traitement cognitif des informations perçues afin d'identifier les possibilités offertes à l'agent en termes d'occupation de l'espace. Ce processus de traitement permet de vérifier que l'affordance perçue correspond réellement à une action possible. Ceci est effectué à l'aide d'une fonction de gain, notée, qui permet d'évaluer une possibilité qui correspond au début à un intervalle virtuel et déterminer si elle peut correspondre à une action à envisager. L'agent évalue les intervalles identifiés. S'il estime avoir un gain en empruntant la voie correspondante à l'intervalle, il affecte cette possibilité à la liste de ses affordances.

La perception produit une représentation ego-centrée de l'environnement, notée $R E E_{\mathrm{ai}}$, construite par l'agent $a_{\mathrm{i}}$ et composée par les affordances identifiées et l'ensemble des caractéristiques de ces dernières noté $P$. Chaque $V V_{1}$ est caractérisée par un ensemble de propriétés donné par $P_{\mathrm{j}}=\left\{p_{1}, p_{2}, \ldots, p_{\mathrm{qj}}\right\}$. Cette représentation correspond à une représentation mentale explicite de la situation, laquelle est propre à chaque agent. Pour sa prise de décision, l'agent construit sa représentation de l'environnement et évalue sa liste des affordances identifiées. Parmi celles-ci, ce dernier choisit la meilleure.

Notons que les ensembles correspondant à chaque représentation ego-centrée dépendent $R E E_{\mathrm{ai}}$ de l'agent qui effectue le raisonnement. Les détails de ce processus ainsi que les différents ensembles introduits sont présentés dans les sections suivantes.

\subsubsection{Définition des affordances}

Une affordance correspond à une possibilité d'occupation de l'espace au sol. Elle dépend du contexte mais aussi des capacités physiques et mentales de l'agent. Plus concrètement, elle correspond à une voie virtuelle permettant in fine une possibilité d'occuper un emplacement sur la chaussée.

Pour notre modèle, nous avons choisi de conserver cette notion de voies de circulation pour le déplacement des agents et la structuration de l'espace. Par conséquent, les affordances correspondent aux voies virtuelles offrant des possibilités d'occupation de l'espace. Elles ont des caractéristiques permettant de les évaluer par la suite et faire un choix de déplacement.

Dans le cadre de notre application, nous postulons que le nombre d'affordances ne peut pas dépasser 5. Les affordances correspondent aux possibilités suivantes :

- Rester sur sa propre voie

- Aller vers la gauche pour atteindre une voie adjacente

- Aller vers la droite pour atteindre une voie adjacente
- Aller vers la gauche pour atteindre un espace plus loin à gauche

- Aller vers la droite pour atteindre un espace plus loin à droite

En effet, dans une situation routière donnée, et en situation d'interaction, le conducteur a le choix entre rester sur sa voie et s'adapter à la contrainte de vitesses des autres véhicules le précédant, et changer de voie à gauche ou à droite. Ces trois possibilités correspondent ainsi aux trois premières affordances précitées. De plus, un conducteur a besoin d'avoir de l'information plus loin que l'environnement adjacent afin de pouvoir détecter des options plus favorables non atteignables directement mais par itérations de manœuvres. Par conséquent, les deux dernières possibilités représentent l'existence d'opportunités « atteignables » vers la droite ou vers la gauche, au-delà des voies adjacentes. Ces voies ne sont pas nécessairement doublement adjacentes (adjacentes aux voies adjacentes). Elles désignent des voies qui sont atteignables par une succession de manœuvres de changement de voies. Elles permettent, par exemple, d'indiquer une option favorable atteignable au prix de changements de voies parfois défavorables. Ces alternatives nous permettent de prendre en compte non seulement l'environnement adjacent mais aussi des possibilités plus éloignées permettant d'avoir une perception plus étendue de l'environnement des agents. Le changement de voie peut donc être une solution immédiate à la contrainte, ou une étape transitoire vers cet objectif, et ce dans le cas où le conducteur essaye d'atteindre une voie « favorable » en empruntant des voies «non favorables ».

L'ensemble $A f f_{\mathrm{i}}=\left\{V V_{1}, V V_{2}, \ldots, V V_{1}\right\}$, avec $l \leq 5$, donne la liste des affordances identifiées par l'agent, où chaque affordance correspond à une voie virtuelle possible. La détermination des affordances est effectuée à travers un parcours de l'espace autour de l'agent en partant de l'environnement le plus proche et en allant vers le plus éloigné.

\subsubsection{Mécanisme d'identification des affordances}

Nous présentons dans ce qui suit les différentes étapes permettant à l'agent de déterminer la liste des affordances. À partir de la liste des agents perçus, ordonnés selon leur position latérale à partir du bord droit de la route jusqu'au bord gauche, l'agent identifie l'ensemble des intervalles virtuels $I V=\left\{i v_{1}, i v_{2}, \ldots, i v_{\mathrm{m}}\right\}$. Un intervalle virtuel est caractérisé par une largeur et est défini soit par l'espace disponible entre deux véhicules ou un véhicule et le bord de la route, soit par l'espace occupé par un véhicule. À cette étape, il peut y avoir des intervalles qui se chevauchent ou un intervalle inclus dans un autre, dû à la position des 
véhicules sur la chaussée. Afin d'identifier les intervalles libres de ceux qui sont occupés, nous allons nous inspirer du modèle des intervalles d'Allen [43] pour raisonner sur la construction des intervalles virtuels.

Allen [43] a défini un modèle permettant de décrire tous les positionnements relatifs possibles entre deux intervalles temporels. Soient deux intervalles $X$ et $Y$, les relations mentionnées par Allen permettant de raisonner sur les intervalles sont données par la figure 4 .

Dans notre modèle, nous adaptons ces relations à une représentation spatiale afin de permettre à l'agent d'identifier et de restructurer les intervalles virtuels. Ces relations sont utiles pour identifier les relations de conflit qui peuvent exister entre les intervalles spatiaux. Par exemple, deux intervalles construits chacun à partir d'une largeur d'un véhicule qui se chevauchent doivent être fusionnés en un seul intervalle occupé. Les intervalles libres et occupés résultants doivent couvrir la largeur de la route. Par conséquent, deux intervalles adjacents doivent en final avoir une relation « rencontre ».

L'agent commence par identifier les intervalles formés par les espaces libres et occupés par des véhicules. Chaque intervalle $X$ est caractérisé par une largeur donnée par la fonction $\operatorname{larg}(X)$. De plus chaque intervalle a une position de début donnée par $p d(X)$ et une position de fin donnée par $p f(X)$. Pour les intervalles occupés, nous définissons une distance à l'obstacle entre l'agent calculant les intervalles et le véhicule occupant l'intervalle. Cette distance est estimée par $\operatorname{dist}\left(a_{i}, X\right)$ (Figure 5).

Sur la base de ces relations, l'agent identifie les intervalles qui peuvent poser problème. Ces derniers correspondent aux cas suivants :

- un intervalle chevauche un autre,

- un intervalle contient un autre,

- un intervalle débute avec un autre,

- un intervalle se termine avec un autre.

Dans la suite, nous ne développons pas les relations inverses qui sont définies de manière équivalente. Si deux intervalles $X$ et $Y$ se chevauchent, l'agent doit les fusionner pour en considérer un seul :

$$
\begin{aligned}
(X d Y) & \wedge\left(\operatorname{larg}\left(a_{i}\right)>(p f(Y)-p f(X))\right) \\
& \rightarrow \text { fusionner }(X, Y)
\end{aligned}
$$

Si un intervalle $X$ contient un intervalle $Y$ alors l'agent conserve dans l'ensemble des intervalles, celui qui a la largeur la plus grande :

$$
(X d Y) \wedge(\operatorname{larg}(X)<(\operatorname{larg}(Y)) \rightarrow I V \backslash\{X\}
$$

Si un intervalle $X$ commence un intervalle $Y$, la règle est :

$$
(X s Y) \wedge(\operatorname{larg}(X)<(\operatorname{larg}(Y)) \rightarrow I V \backslash\{X\}
$$

De même, si un intervalle $X$ finit un intervalle $Y$, la règle est :

$$
(X f Y) \wedge(\operatorname{larg}(X)<(\operatorname{larg}(Y)) \rightarrow I V \backslash\{X\}
$$

Enfin, si un intervalle $X$ est égal à un intervalle $Y$, l'agent garde l'intervalle le plus proche de lui :

$$
(X=Y) \wedge\left(\operatorname{dist}\left(a_{i}, X\right)<\operatorname{dist}\left(a_{i}, Y\right)\right) \rightarrow I V \backslash\{Y\}
$$

Pour illustrer ce raisonnement, nous reprenons l'exemple de la figure 5. Dans cet exemple, nous avons un agent $a_{2}$ mal garé, un deuxième agent $a_{3}$ se trouvant sur la voie 1 de circulation et un deux-roues motorisé $a_{1}$. Le raisonnement est effectué par l'agent. Dans ce cas de figure, il y a deux intervalles, $X$ et $Y$, qui se chevauchent. L'agent applique la première règle de fusion d'intervalles pour résoudre le conflit de représentation spatiale. Étant donné que l'espace libre $(p f(Y)-p f(X))$ n'est pas suffisant pour permettre au deux-roues motorisé de poursuivre sa trajectoire, l'agent $a_{1}$ considère que les intervalles $X$ et $Y$ correspondent, au final, à un seul intervalle occupé par les deux véhicules et fusionne les deux intervalles pour en conserver un seul.

Toutes ces règles permettent à l'agent de raisonner sur les intervalles afin d'identifier les sources de conflit et restructurer les espaces libres et occupés. À partir de la liste des intervalles virtuels résultant du raisonnement précédent, l'agent identifie la liste des affordances en parcourant les intervalles du plus proche au plus éloigné (selon la position latérale de l'agent et les positions de début et de fin de chaque intervalle). Pour ce faire, il commence par identifier et caractériser son environnement immédiat (sa propre voie) ensuite son environnement adjacent (les voies adjacentes à gauche et à droite) et enfin son environnement lointain ou non adjacent (les voies non adjacentes à gauche et à droite).

Environnement immédiat. L'agent parcourt l'ensemble $I V$ et identifie l'intervalle $i v_{\mathrm{k}}$ auquel il appartient d'après sa position latérale. Cet intervalle se transforme en une voie $l_{\mathrm{k}}$ en renseignant les paramètres cités précédemment tels que la longueur (la distance à l'obstacle sur cette voie), la vitesse, la densité, etc. Cette voie est ensuite ajoutée à l'ensemble des affordances $A f f$, elle correspond à l'action « rester sur sa propre voie et aller tout droit ».

Environnement adjacent. À partir de son environnement immédiat (sa propre voie), l'agent parcourt l'espace vers la gauche et vers la droite afin d'identifier et caractériser les affordances possibles. L'agent commence par les intervalles 


\begin{tabular}{|c|c|c|c|c|c|c|}
\hline Précède & Chevauche & Rencontre & Dans & Débute & Termine & Égal \\
\hline $\mathrm{x}$ & $\mathrm{x}$ & $\mathrm{x}$ & $\stackrel{x}{x}$ & $\mathrm{x}$ & $\mathrm{x}$ & \multirow{5}{*}{$\frac{\mathrm{X}}{\longmapsto_{\mathrm{Y}}}$} \\
\hline$\longmapsto$ & $\bar{Y}$ & $\mathrm{Y}$ & $\mathrm{Y}$ & $\mathrm{Y}$ & $\bar{Y}$ & \\
\hline $\mathrm{X}<\mathrm{Y}$ & $\mathrm{X} \circ \mathrm{Y}$ & $\mathrm{XmY}$ & $\mathrm{XdY}$ & $\mathrm{X}_{5} \mathrm{Y}$ & $X f Y$ & \\
\hline Suit & chevauché par & Rencontré par & Contient & Débuté par & Terminé par & \\
\hline$\stackrel{\mathrm{Y}}{ } \longmapsto^{\mathrm{X}}$ & $\stackrel{\mathrm{Y}}{\stackrel{\mathrm{x}}{\stackrel{⿱}{\longmapsto}}}$ & $\stackrel{\mathrm{Y}}{\stackrel{\mathrm{x}}{\longmapsto}}$ & $\longmapsto \frac{\mathrm{X}}{\longmapsto}$ & $\stackrel{\mathrm{x}}{\stackrel{\mathrm{Y}}{\longmapsto}}$ & $\stackrel{\mathrm{X}}{\longmapsto}$ & \\
\hline$Y>X$ & $\mathrm{Y}$ oi $\mathrm{X}$ & $Y \operatorname{mix}$ & $Y \operatorname{diX}$ & $\mathrm{Y} \operatorname{si} \mathrm{X}$ & $Y$ fix & $X=Y$ \\
\hline
\end{tabular}

Fig. 4 Les relations d'Allen entre deux intervalles.

adjacents à son propre intervalle. Ces intervalles sont donnés par ceux qui ont une relation « rencontre » avec l'intervalle de l'agent, l'un à gauche et l'autre à droite. Ensuite, l'agent évalue chacun des deux intervalles identifiés. S'il estime avoir un gain en empruntant la voie correspondante à l'intervalle, il affecte cette possibilité à la liste de ses affordances. Sinon, il fusionne cet intervalle avec le suivant (s'il n'y a pas de marquage physique passant par cet intervalle) ou il fusionne jusqu'au marquage. L'agent ajoute ensuite cette nouvelle voie à sa liste d'affordances.

Le mécanisme d'évaluation est fondé sur les propriétés des voies identifiées précédemment. Chaque agent a le choix entre deux actions : rester sur sa voie ou changer de voie. Cette évaluation est effectuée à travers une fonction qui compare la vitesse courante de l'agent et la vitesse estimée qu'il pourrait avoir sur la voie cible. Cette fonction est donnée par la différence entre les deux vitesses pour un agent $a_{\mathrm{i}}^{3}$ :

$$
\operatorname{gain}\left(v c_{a i}\right)=\tau * v_{a i}\left(l_{k}\right)-v c_{a i}
$$

Avec $l_{\mathrm{k}}$ représente la voie $k, v_{\mathrm{ai}}\left(l_{\mathrm{k}}\right)$, correspond à la vitesse estimée sur la voie $l_{\mathrm{k}}, v c_{\mathrm{ai}}$ est la vitesse courante de l'agent $a_{\mathrm{i}}$ et $\tau$ compris entre 0 et 1 , correspond à l'acceptabilité sociale de la pratique de faufilement qui diffère selon le type du véhicule (deux-roues, voitures, bus, etc.). Plus précisément, $v_{a i}\left(l_{\mathrm{k}}\right)^{4}$ dépend des paramètres suivants :

- $f_{a i}\left(l_{\mathrm{k}}\right)$ : un paramètre lié aux caractéristiques de flux de la voie $l_{\mathrm{k}}$ et qui dépend de la densité de la voie et de la vitesse moyenne,

- $g_{a i}\left(l_{\mathrm{k}}\right)$ : un paramètre lié à l'effet paroi de la voie $l_{\mathrm{k}}$ et qui dépend de la proximité des parois et de leur stabilité en termes de vitesses,

- $h_{a i}\left(l_{\mathrm{k}}\right):$ un paramètre lié aux caractéristiques individuelles de chaque agent et qui traduit sa distance par rapport à la réglementation (d'un comportement normatif à un comportement non normatif).
N'ayant pas trouvé d'études détaillées concernant les valeurs que peuvent prendre ces paramètres, nous avons tenté de les attribuer d'une manière subjective et empirique afin d'avoir une tendance au niveau des résultats. Il est difficile de trouver dans la littérature en psychologie de la conduite ou dans les travaux en simulation multi-agents de trafic des références concernant les valeurs que peuvent prendre des paramètres tels que par exemple l'effet paroi d'une voie. Afin de pouvoir identifier les ordres de grandeur, nous nous sommes fondés sur des études qui donnent des indications concernant les différentiels de vitesses que l'on peut observer lors de remontées de files [44]. Nous proposons l'expression suivante :

$$
v_{a i}\left(l_{k}\right)=\left(a^{*} f_{a i}\left(l_{k}\right)+b^{*} \text { vitMoyParoi }\right)^{*} g_{a i}\left(l_{k}\right)^{*} h_{a i}\left(l_{k}\right)
$$

Les trois paramètres sont définis : $f_{a i}\left(l_{k}\right)=A * \min \left(v_{\text {desir }}\right.$, vitMoy) $+B * v_{\text {desir }}$;

$$
\begin{aligned}
g_{a_{i}}\left(l_{k}\right)= & \frac{\text { distance }}{\text { diffVitParois }+1} \text { et } h_{a_{i}}\left(l_{k}\right) \\
& =\frac{2 * \text { dRegle }+ \text { dTrafic }}{100} .
\end{aligned}
$$

avec :

$$
\begin{aligned}
A= & e \frac{\text { dist Obstacle }}{\text { distPerception }} ; B=1-A ; a \\
& =e \frac{\text { distance }}{\text { distanceSécurité }} ; b=1-a
\end{aligned}
$$

- distance: distance séparant le véhicule de ses parois

- distanceSécurité : distance de "sécurité » de l'agent en latéral (espace accepté en fonction de la vitesse pratiquée, et de la qualité des parois)

- diffVitParois : différentiel de vitesse entre la paroi gauche et droite

- distObstacle : distance à l'obstacle devant le véhicule de l'agent 
- distPerception : distance de perception de l'agent (qui dépend de sa vitesse)

- dRegle : caractéristique de l'agent précisant le niveau de respect de la réglementation de l'agent (comportement normatif ou non-normatif)

- dTrafic : caractéristique de l'agent précisant la facilité de manœuvrer dans le trafic de l'agent

- $v_{\text {desir }}$ : vitesse désirée de l'agent, c'est-à-dire celle qui pratiquerait s'il n'était pas «gêné » par le véhicule devant lui

- vitMoy : vitesse moyenne de la voie en question, dans l'environnement de l'agent

- vitMoyParoi : vitesse moyenne des parois, dans l'environnement de l'agent

La fonction de gain est positive quand la voie cible présente un intérêt pour l'agent en termes de vitesse. Cette estimation prend en compte les caractéristiques des voies (largeur, longueur, etc.), les caractéristiques des parois (stabilité, proximité) et les caractéristiques individuelles des agents, notamment la distance par rapport à la norme. Une voie virtuelle peut ne pas présenter le même intérêt selon que l'agent ait un comportement normatif ou pas.

Environnement lointain. Pour les voies non immédiatement adjacentes, nous proposons d'évaluer, en parcourant la chaussée de la voie immédiatement adjacente vers le bord de la route (latéralement à droite et à gauche) et de retenir la première voie (virtuelle ou non) dont les caractéristiques sont "meilleures » que la voie courante (le parcours s'effectue sur l'ensemble). Par « meilleure » nous entendons une voie qui permettrait à l'agent d'espérer un gain selon des critères qui lui sont propres (dans notre cas, un gain en vitesse) et qui dépend des caractéristiques individuelles.

Le résultat de cette étape est l'ensemble des affordances correspondant à toutes les opportunités identifiées par l'agent.

\subsubsection{Représentation ego-centrée fondée sur les affordances}

Après avoir identifié la liste des affordances, l'agent se construit une représentation ego-centrée de l'environnement fondée sur les affordances détectées. Plus formellement, la représentation ego-centrée de l'environnement d'un agent $a_{\mathrm{i}}$ (notée $R E E_{a i}$ ) est définie par le tuple $<A, A f f, R>$ :

- $A=\left\{a_{1}, a_{2}, \ldots, a_{n}\right\}$ est un sous-ensemble de tous les agents perçus par $a_{1}$

- Aff $=\left\{V V_{1}, V V_{2}, \ldots, V V_{1}\right\}$, avec $l \leq 5$, désigne l'ensemble des affordances identifiées. Ces affordances peuvent être différentes des voies physiques initialement définies par le marquage au sol,

$-R=\left\{r_{a i}\left(w_{\mathrm{k}}\right) / w_{\mathrm{k}} \in A \mathrm{U}\right.$ Aff $\}$ désigne l'ensemble des relations qui relient les objets et les agents. Chaque $r_{\mathrm{ai}}\left(w_{\mathrm{k}}\right)$ établit une relation binaire entre l'agent $a_{\mathrm{i}}$ et chaque agent $a_{k}$ de $A$ traduisant la relation spatiale entre les deux agents (à sa droite, à sa gauche, devant, derrière, etc.) ou une relation binaire entre l'agent et chaque voie virtuelle $V V_{k}$ de $A f f$ traduisant la relation entre l'agent et la voie considérée (voie de l'agent, voie adjacente à gauche, voie adjacente à droite, etc.).

Les voies virtuelles identifiées sont caractérisées par les propriétés citées précédemment. Chaque $P_{\mathrm{i}}$ est l'ensemble des propriétés de la voie $i$.

\subsection{Décision et action}

La représentation ego-centrée de l'environnement permet à l'agent de prendre une décision en choisissant l'affordance optimale. Le résultat du mécanisme de décision est une voie virtuelle possible avec un certain nombre de critères tels que la largeur, la position au niveau de l'espace routier, la vitesse de la voie, les vitesses des parois. Ces critères permettent d'identifier la position latérale future de l'agent et une vitesse latérale possible.

Grâce à la généralisation de l'usage des voies virtuelles ainsi qu'à l'enrichissement des caractéristiques liées aux voies, nous prévoyons que l'alternative d'opter pour une voie virtuelle d'inter-files n'est pas systématique et particulièrement pour les véhicules automobiles, les poids-lourds, pour lesquels la «tolérance » liée à l'usage de ce type de voies est faible de même que le gain en termes de temps de parcours. Pour ces derniers usagers, elle est plutôt favorisée en cas d'événements spécifiques (par exemple, la présence d'un véhicule mal garé ou un véhicule d'urgence). La pratique de l'inter-file est plutôt réservée aux deux-roues du fait d'une tolérance et d'un gain important en termes de temps de parcours. La solution que nous proposons devrait aussi permettre d'élargir le domaine de validité du modèle pour des situations de réseaux routiers avec un nombre de voies important.

À partir de cette représentation ego-centrée de l'environnement fondée sur les affordances, l'agent décide quelle affordance choisir. Pour ce faire, il calcule un score pour chaque affordance de l'ensemble aff. Ce score peut quantifier « l'attractivité » (strength ou attractiveness) relative de toutes les affordances, en se fondant sur les buts désirés de l'agent et sa propre représentation de l'environnement. Ce score est défini par :

$$
F_{a f f}\left(V V_{i}\right)=f\left(p_{1 i}, p_{2 i}, \ldots, p_{\mathrm{qi}}, \text { Buts }\right)
$$


où la fonction $f$ est définie de telle manière que $F_{\text {aff }}(V V)$ fournit une valeur numérique indiquant l'attractivité (une utilité espérée) d'une affordance donnée. Elle est donnée par une somme pondérée des propriétés des affordances $p_{1 i}, p_{2 i}, \ldots, p_{\mathrm{q} i}$ telles que la largeur de la voie, la vitesse moyenne, etc.

Le choix de l'affordance optimale est effectué à travers une évaluation de toutes les affordances identifiées afin de déterminer la meilleure. Par meilleure, nous sous-entendons une affordance offrant de meilleures caractéristiques que la voie courante de l'agent en termes de vitesse de passage. Le résultat final du système de décision est l'affordance $V V_{\mathrm{i}}$ associée à la valeur optimale $F_{\mathrm{aff}}\left(V V_{\mathrm{i}}\right)$ par maximisation de la fonction $f$ :

$$
V V_{\text {optimale }}=\arg \max _{i=1,5} F_{a f f}\left(V V_{i}\right)
$$

Après avoir choisi une affordance, les agents calculent la vitesse à laquelle ils vont se déplacer. Pour cela, ces derniers identifient à partir du trafic environnant (agents et objets routiers géographiquement proches), la contrainte la plus exigeante (c'est-à-dire, celle entraînant la décélération la plus importante). La vitesse est donnée par l'expression suivante [45] :

$$
\begin{aligned}
& V(\Delta x) \\
& =\min \left(\frac{\Delta x}{T I V_{\min } \frac{d T r a f i c}{100}+T I V_{\max }\left(1-\frac{d T \text { rafic }}{100}\right)}, v_{\text {desir }}\right)
\end{aligned}
$$

avec $T I V$ : le temps inter-véhiculaire.

Cette expression exprime également le fait que si le véhicule n'est pas en situation contrainte, il peut circuler à sa vitesse désirée.

\section{Validation}

Nous avons implémenté notre modèle dans l'outil de simulation de trafic, ArchiSim [4]. La validation de notre approche est faite à deux niveaux : une première évaluation du comportement dans une situation critique, le carrefour ; et une seconde fondée sur la simulation d'un circuit réel et la comparaison entre les données de trafic réelles et simulées.

La première partie de notre évaluation consiste en une évaluation visuelle des comportements simulés des conducteurs. Pour évaluer les comportements individuels des agents en termes de positionnement dans l'espace routier, nous utilisons différents scénarii permettant de placer les agents dans des situations de trafic appropriées pour observer le comportement désiré. Nous proposons une validation se basant sur le comportement habituel de déplacement des deux-roues $[42,46]$. Le scénario proposé dans ce cadre, vise à reproduire un comportement que nous observons souvent dans la réalité : une remontée de file au niveau d'un carrefour avec tourner à gauche et à droite. Dans une deuxième partie, nous validons notre modèle d'un point de vue macroscopique en comparant les données réelles collectées sur un circuit et les données de simulation.

\subsection{Situation d'un carrefour avec tourner à gauche et à droite}

Ce scénario a pour objectif d'évaluer une situation au niveau d'un carrefour avec des véhicules qui tournent à droite, d'autres qui tournent à gauche et un véhicule qui se déplace tout droit. Ce scénario vise à reproduire un comportement que nous observons souvent au niveau des carrefours. Généralement, les véhicules qui tournent à droite se serrent plus vers la droite, ceux qui tournent à gauche se serrent vers le bord gauche de la route permettant ainsi aux véhicules allant tout droit de passer. Les figures 6 et 7 présentent une illustration du scénario destiné à évaluer les capacités d'adaptation des agents en termes d'occupation de l'espace. Sur la figure 6, les agents se déplacent en se référant aux voies physiques données par le marquage au sol. Nous nous intéressons au comportement que peut avoir le véhicule 0 . Les véhicules 1,3 et 5 vont tourner à droite. Les véhicules 2, 4 et 6 vont tourner à gauche. Nous constatons que le véhicule 0 reste bloqué derrière la file de véhicules qui tournent à droite. Ce n'est que lorsque les véhicules 1,3 et 5 ont tourné à droite, que le véhicule 0 traverse le carrefour (pas de temps 671).

La figure 7 présente le même scénario avec des agents dotés de notre modèle. Le mobile 0 se construit une représentation de la situation. Étant donné que les véhicules 1,3 et 5 tournent à droite, ils se serrent vers le bord droit de la route. Les véhicules 2,4 et 6 observent le même comportement mais à gauche. Nous constatons que dès le pas de temps $t=3$, le mobile 0 met son clignotant et commence à déboîter sur une voie virtuelle entre les deux files de véhicules. À $t=159$, le mobile a déjà traversé le carrefour alors qu'au même pas de temps, il était encore bloqué derrière la file de véhicules dans la première simulation (Figure 6).

Le mobile 0 se construit une représentation ego-centrée de la situation fondée sur les affordances. Il identifie ainsi trois affordances :

- la voie la plus à droite, formée par les véhicules 1,3 et 5,

- la voie la plus à gauche, formée par les véhicules 2,4 et 6 ,

- la voie qui émerge au milieu entre les deux files de véhicules. 


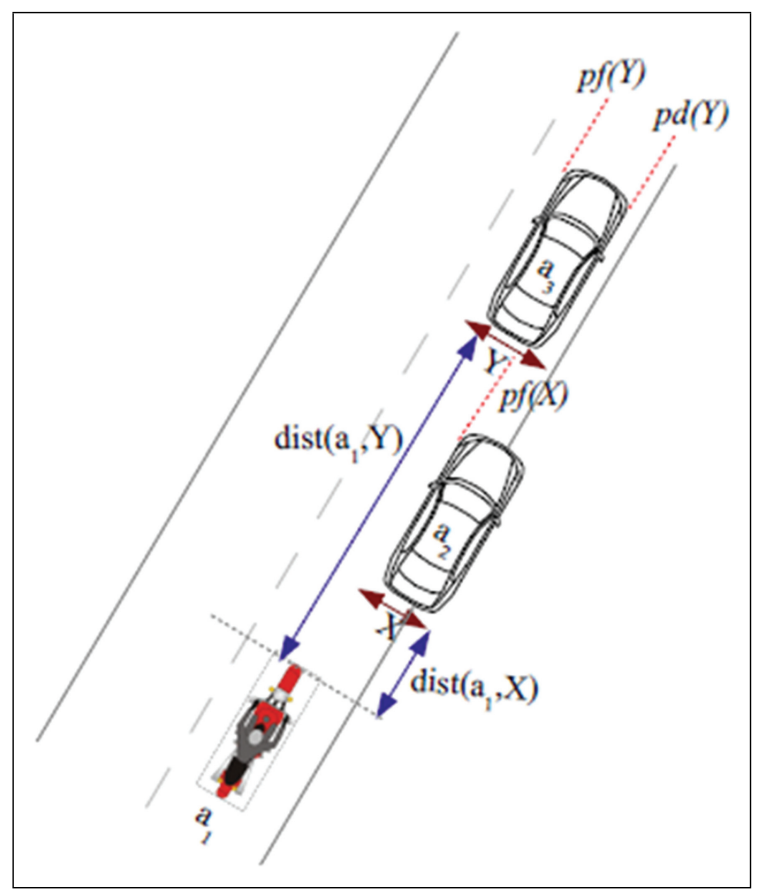

Fig. 5 Situation de chevauchement de deux intervalles

Par conséquent, la représentation ego-centrée de l'environnement du mobile 0 est composée de ces trois affordances. Afin de prendre une décision, le mobile évalue ces dernières et opte pour la meilleure, c'est-à-dire, la voie virtuelle entre les deux files de véhicules. Cette voie présente de meilleures caractéristiques en termes de vitesse et permet au mobile de débloquer sa situation et de traverser le carrefour.

\section{2. Évaluation du trafic obtenu}

Pour évaluer d'un point de vue macroscopique le trafic obtenu, nous avons choisi un circuit réel de $31 \mathrm{~km}$ de long avec des routes départementales, nationales et des autoroutes. Notons que pour la problématique de l'occupation de l'espace et plus particulièrement pour les phénomènes d'occupation de l'espace tels que nous pouvons les observer dans la réalité, il existe très peu de données permettant de valider notre modèle. Cependant, nous utilisons des données issues d'une étude effectuée par l'Agence de l'environnement et de la maîtrise de l'énergie (Ademe) [47].

\subsubsection{Simulation d'un circuit réel}

Le circuit utilisé correspond à un itinéraire de $31 \mathrm{~km}$ de long. Le départ avait lieu de Linas-Monthléry (91) et l'arrivée était au musée d'Orsay (75007). L'étude effectuée par l'Ademe avait pour but de comparer la consommation et les émissions polluantes des deux-roues motorisés à celles des voitures.

La méthode de collecte des données de trafic consiste à faire des enregistrements simultanés, sur route, dans des conditions encadrées pour un scooter $125 \mathrm{~cm}^{3}$, une moto de $600 \mathrm{~cm}^{3}$ et une voiture urbaine. Les conducteurs des deux-roues motorisés étaient expérimentés. Leur conduite devait être raisonnée, c'est-à-dire avec le respect des limitations de vitesse et l'autorisation pour les deux-roues de remonter les files si les files de voitures étaient au ralenti. Le trajet type est du domicile-travail de la banlieue de Paris au centre de Paris. Ces enregistrements nous fournissent des données telles que par exemple, la vitesse moyenne, le temps de parcours et le nombre d'arrêts.

\subsubsection{Protocole des expérimentations}

Nous avons utilisé ArchiSim pour reproduire le circuit utilisé par l'Ademe. Pour cette expérimentation, nous avons reproduit $23 \mathrm{~km}$ du circuit initial. Nous avons utilisé les mêmes caractéristiques physiques (routes départementales, routes nationales, autoroutes), les mêmes feux tricolores et marquages au sol. Nous avons aussi placé dans la simulation des capteurs virtuels positionnés aux mêmes points kilométriques que dans la réalité. Nous avons ensuite généré une demande de trafic hétérogène comme suit :

- Pour chaque véhicule, la position et l'instant de génération ont été définis en utilisant une loi de Poisson.

- La vitesse désirée de chaque véhicule a été générée selon une loi Gaussienne.

- La vitesse initiale de chaque véhicule est définie comme étant le minimum entre la vitesse désirée et la vitesse du véhicule juste devant.

- La proportion des deux-roues motorisés, des camions et des bus dépend du nombre total des véhicules.

Nous avons par la suite ajouté une voiture et un deux-roues motorisé dans les mêmes conditions de vitesse et de position.

\subsubsection{Comparaison entre les données réelles et les données simulées}

Les simulations ont été réalisées dans deux situations expérimentales différentes. Dans la première situation, les agents ont le comportement de référence qui correspond au modèle d'ArchiSim sans les voies virtuelles : les agents se positionnent selon les voies physiques données par le marquage au sol. La deuxième situation correspond aux agents dotés du modèle fondé sur les affordances. Nous 


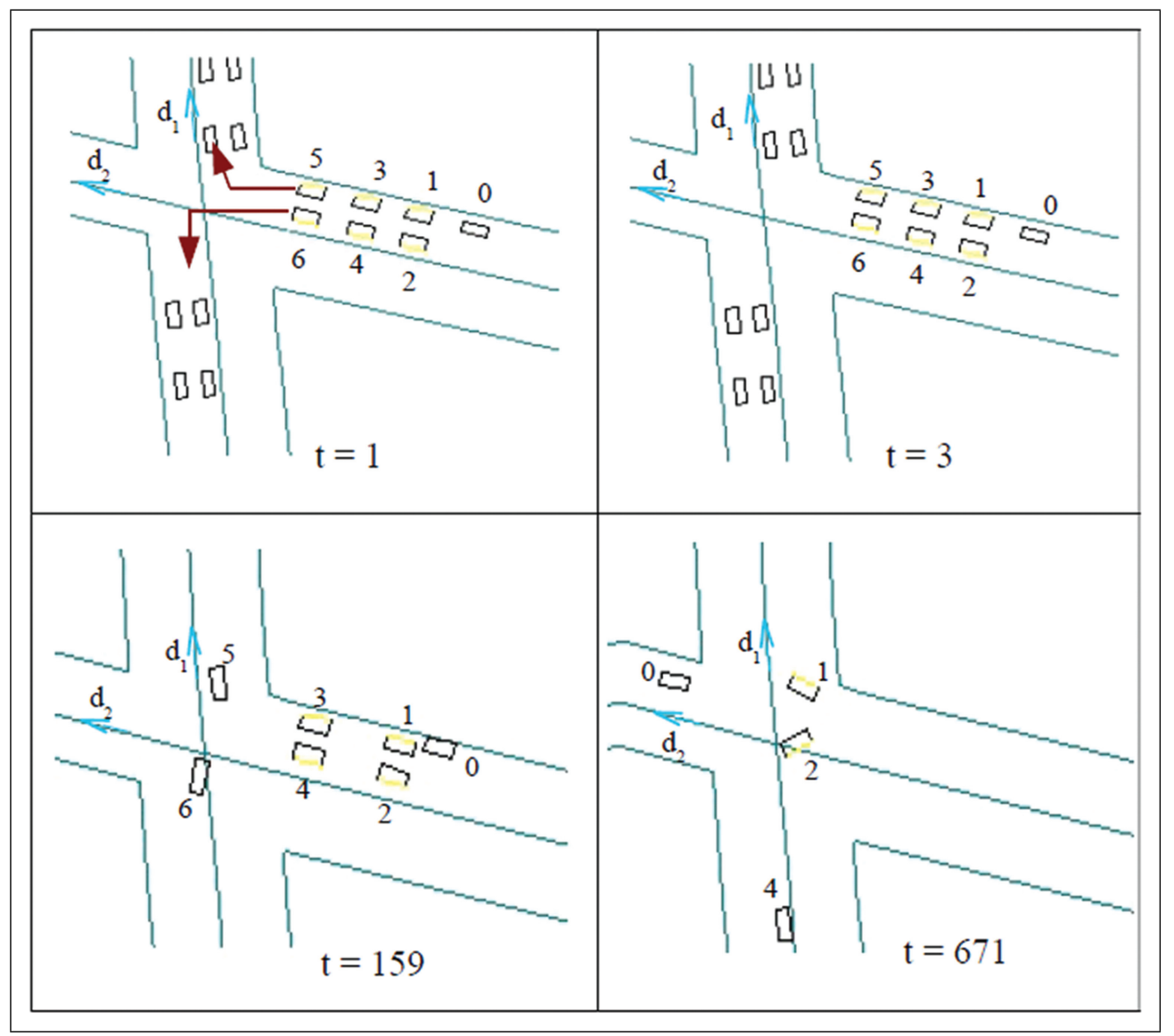

Fig. 6 Simulation sans les voies virtuelles au niveau d'un carrefour

comparons d'abord les résultats de notre modèle avec ceux du modèle de référence dans le but d'évaluer l'impact de notre modèle sur le trafic. Ensuite, nous comparons nos résultats aux données réelles. Les données réelles et les résultats des simulations (avec notre modèle et le modèle de référence) sont résumés dans le tableau 1 .

Nous nous intéressons au comportement d'une voiture et d'un 2RM générés avec les mêmes caractéristiques afin d'étudier l'impact de notre modèle. Les données réelles montrent que le 2RM met la moitié du temps de parcours de la voiture pour un même trajet. Nous ne retrouvons pas cette même tendance pour les résultats de simulation du modèle de référence où les temps de parcours entre moto et voiture sont très proches. Ce dernier résultat est dû au fait, que dans le modèle de référence, le comportement de déplacement est le même pour les voitures et les 2RM. Tous les usagers de la route utilisent les voies physiques données par le marquage au sol.

Nous comparons les résultats de simulation de notre modèle avec ceux du modèle de référence. La différence entre les temps de parcours de la voiture et de la moto a été améliorée par rapport au modèle de référence, la moto prend moins de temps pour effectuer le même trajet. Par conséquent, la vitesse moyenne de la moto est supérieure à celle de la voiture. Ainsi, le modèle fondé sur les affordances permet à l'agent conduisant la moto d'identifier les voies virtuelles offertes par l'environnement. En raison de leur taille, les $2 \mathrm{RM}$ ont tendance à utiliser les voies virtuelles plus que les autres types de véhicules. Leur temps de parcours est inférieur à celui des voitures.

Notons que l'on observe les mêmes tendances, lorsque l'on compare les résultats de notre modèle et les données réelles. L'écart entre les résultats de simulation et les données réelles est dû à un problème de calibration de la simulation. Nous ne disposons en effet d'aucune donnée permettant d'ajuster précisément les débits ou les vitesses des autres véhicules. De plus, notre modèle ne détériore pas les résultats obtenus par rapport aux travaux existants, notamment les travaux de Bonte et al. [24].

Nous pouvons ajouter que notre modèle prend en compte le fait que la pratique du faufilement est mieux tolérée pour les deux-roues motorisés que pour les autres types de véhicules. D'autres travaux que nous avons effectués [46] montrent que le conducteur de la voiture choisit la solution d'une voie virtuelle uniquement s'il a un comportement non normatif (cas extrême). Le choix des 


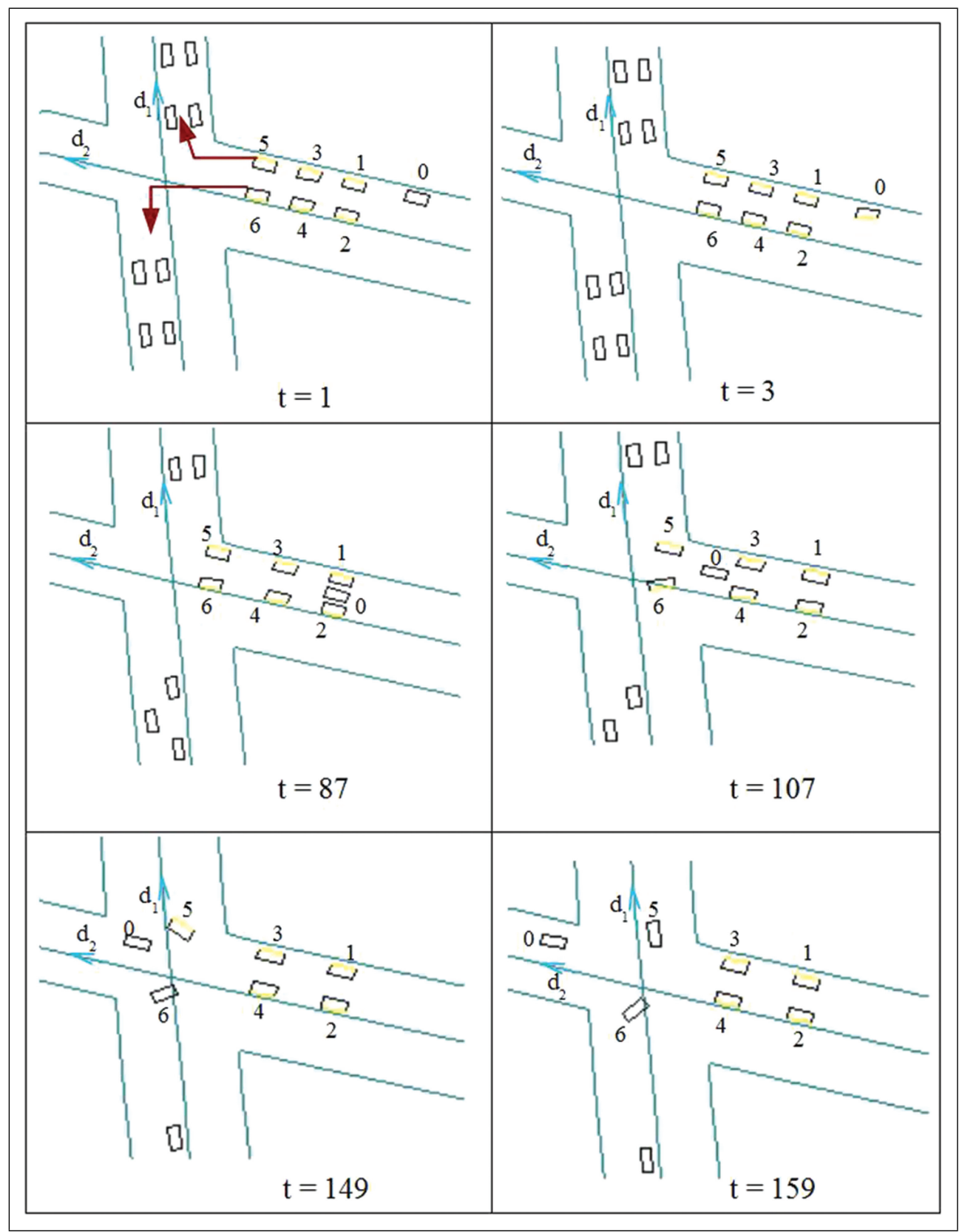

Fig. 7 Simulation avec le modèle à base d'affordances au niveau d'un carrefour

voies virtuelles n'est pas systématique, il dépend des caractéristiques des voies ainsi que des caractéristiques du véhicule (la taille par exemple) et des caractéristiques individuelles des agents (la distance par rapport à la réglementation). Notre modèle est générique dans le sens où il n'est pas spécifique à un type particulier de conducteur. L'hétérogénéité des comportements résulte des différents contextes de conduite et des caractéristiques individuelles des conducteurs couplés à des règles génériques.

\section{Conclusion}

Nous nous intéressons à la simulation de phénomènes de trafic routier à travers une approche comportementale fondée sur les SMA. Les travaux envisagés ont pour but d'étendre le champ de validité des simulations de trafic en milieux urbain et périurbain, avec une meilleure prise en compte de l'hétérogénéité des véhicules et des comportements des conducteurs en termes de positionnement sur les voies et d'occupation de l'espace. 


\begin{tabular}{lllll}
\hline \multicolumn{1}{l}{ Tableau 1 Données réelles et données de simulation pour une route de $23 \mathrm{~km}$} & & \\
\hline & Type du véhicule & Temps de parcours & Vitesse moyenne (km/h) & Nombre d'arrêts \\
\hline \multirow{2}{*}{ Données réelles } & Voiture & $1 \mathrm{~h} 03$ min 09sec & 21,85 & 51 \\
& $2 \mathrm{RM}$ & $32 \mathrm{~min} 01 \mathrm{sec}$ & 43,10 & 16 \\
Modèle de référence & Voiture & $54 \mathrm{~min} 21 \mathrm{sec}$ & 25,40 & 141 \\
& $2 \mathrm{RM}$ & $52 \mathrm{~min} 58 \mathrm{sec}$ & 26,05 & 137 \\
Modèle de (Bonte et al. 2006) & Voiture & $57 \mathrm{~min} 06 \mathrm{sec}$ & 24,29 & 77 \\
& $2 \mathrm{RM}$ & $32 \mathrm{~min} 32 \mathrm{sec}$ & 42,64 & 31 \\
Modèle fondé sur les affordances & Voiture & $58 \mathrm{~min} 47 \mathrm{sec}$ & 23,52 & 91 \\
& $2 \mathrm{RM}$ & $32 \mathrm{~min} 10 \mathrm{sec}$ & 43,09 & 35 \\
\end{tabular}

Nous avons proposé, dans ce papier, un nouveau modèle d'agent fondé sur une représentation ego-centrée de l'environnement. Cette représentation permet à l'agent de prendre une décision en termes d'occupation de l'espace au sol. Notre approche est fondée sur le concept des affordances - décrivant la manière avec laquelle un agent peut interagir avec son environnement. Les affordances permettent d'identifier les actions possibles, en termes d'occupation de l'espace, offertes par l'interaction des entités perçues avec l'environnement. Dans le contexte de notre application, une affordance correspond à une voie virtuelle possible.

Nous avons implémenté notre modèle avec l'outil de simulation de trafic ArchiSim. Nous avons validé quelques comportements individuels notamment le faufilement et la remontée de files des 2RM. Dans des situations de trafic plus complexes, nous obtenons les mêmes tendances en évaluant la comparaison entre les résultats obtenus par notre modèle et ceux issus de données réelles. Dans notre modèle, l'écart entre le temps de parcours d'une voiture et d'une moto a été amélioré par rapport aux résultats de simulation du modèle de référence. Le temps de parcours de la moto correspond à presque la moitié de celui de la voiture.

Les résultats obtenus sont prometteurs. Néanmoins, des améliorations sont envisagées. Il est nécessaire de mieux calibrer notre modèle afin de réduire l'écart avec les données réelles. Une autre perspective consiste à décrire un comportement plus coopératif des agents. Ce type de comportement peut être observé dans certaines situations telles que la présence de véhicule d'urgence où les véhicules s'écartent pour laisser passer ce dernier ou parfois dans le cas du faufilement des deux-roues motorisés.

\section{Bibliographie}

1. Bazzan, A, Wahle, J, Klügl, F (1999) Agents in traffic modelling: from reactive to social behavior. In: Burgard, W, Christaller,
T, Cremers Armin, B (eds.) Advances in artificial intelligence. Springer, 1701:303-306

2. Doniec, A, Mandiau, R, Piechowiak, S, Espié, S (2008) Anticipation based on constraint processing in a multi-agent context. Journal of Autonomous Agents and Multi-Agent Systems (JAAMAS) 17(2): 339-361.

3. Vasirani, M, Klugl, F, Camponogara, E, Hattori, H (eds.) (2012) Proceedings of the $7^{\text {th }}$ international workshop on agents in traffic and transportation (att). Valencia, Spain.

4. Espié, S (1995) Archisim, multi-actor parallel architecture for traffic simulation. In: Proceedings of the second world congress on intelligent transport systems, vol. $I V$. Yokohama.

5. Gibson, J (1977) The theory of affordances. In: Shaw, R, Bransford, J (eds.) Perceiving, acting and knowing. Hillsdale, New Jersey, Lawrence Erlbaum and Associates.

6. Minh, C C, Sano, K, Matsumoto, S (2005) The speed, flow and headway analyses of motorcycle traffic. Journal of the Eastern Asia Society for Transportation Studies 6: 1496-1508.

7. Sykes, P (2010) Traffic simulation with paramics. In Barceló, J (ed.) Fundamentals of traffic simulation. Springer, New York, 145:131-171.

8. Casas, J, Ferrer, J, Garcia, D, Perarnau, J, Torday, A (2010) Traffic simulation with aimsun. In: Jaume, B (ed.) Fundamentals of traffic simulation. Springer, New York, 145:173-232.

9. Krajzewicz, D (2010) Traffic simulation with sumo - simulation of urban mobility. In: Barceló, J (ed.) Fundamentals of traffic simulation. Springer, New York, 145:269-293.

10. Fellendorf, M, Vortisch, P (2010) Microscopic traffic flow simulator vissim. Fundamentals of Traffic Simulation. International Series in Operations Research and Management Science 145: 63-93.

11. Hu, T-Y, Liao, T-Y, Chen, L-W, Huang, Y-K, Chiang, M-L (2007) Dynamic simulation assignment model (dynataiwan) under mixed traffic flows for its applications. The proceedings of 86th transportation research board annual meeting. Washington, DC.

12. Ahuja, S (2001) Simulation of driver behaviour in heterogeneous untidy traffic in developing countries. Proceedings of symposium on advanced vehicle technologies, the international mechanical engineering congress and exposition. New York, USA.

13. Lan, LW, Chang, CW (2005) Inhomogeneous cellular automata modeling for mixed trafficwith cars and motorcycles. Journal of advanced transportation 39: 323-349.

14. Lan, LW, Hsu, CC (2006) Formation of spatiotemporal traffic patterns with cellular automaton simulation. Transportation research board 85 th annual meeting. Washington. 
15. Nagel, K, Barrett, CL, Rickert, M (1996) Parallel traffic micro-simulation by cellular automata and application for large scale transportation modeling. Los Alamos National Laboratory, Los Alamos, New Mexico, USA.

16. Spicher, A, Fatès, N, Simonin, O (2009) From reactive multi-agents models to cellular automata - illustration on a diffusion-limited aggregation model. Icaart, pp 422-429.

17. Saad, F (1992) In-depth analysis of interactions between drivers and the road environment: contribution of on-board observations and subsequent verbal report. Proceedings of the 4th workshop of ICTCT. University of Lund.

18. Espié, S (2004) Simulation comportementale et réalité virtuelle: vers une simulation globale du système de trafic 1989-2004. HDR, Université Paris VI.

19. Burmeister, B, Doormann, J, Matylis, G (1997) Agent-oriented traffic simulation. Transactions of the society for computer simulation international 14: 79-86.

20. Hidas, $P$ (2002) Modelling lane changing and merging in microscopic traffic simulation. Transportation Research Part $C$ : Emerging Technologies 5-6: 351-371.

21. El Hadouaj, S (2004) Conception de comportements de résolution de conflits et de coordination: Application à une simulation multiagent du trafic routier. Thèse de doctorat, Université Paris VI.

22. Kesting, A, Treiber, M, Helbing, D (2009) Agents for traffic simulation. Multi-Agent Systems: Simulation and Applications, pp 325-356.

23. Dai, J, Li, X (2010) Multi-agent systems for simulating traffic behaviors. Chinese Science Bulletin 55: 293-300.

24. Bonte, L, Espié, S, Mathieu, P (2006) Modélisation et simulation des usagers deux-roues motorisés dans archisim. Actes des $14^{\mathrm{e}}$ journées francophones sur les systèmes multiagents (JFSMA'06), pp 31-44.

25. Lee, T, Polak, J, Bell, M (2009) New approach to modeling mixed traffic containing motorcycles in urban areas. Transportation Research Record 2140: 195-205.

26. Gipps, PG (1981) A behavioural car-following model for computer simulation. Transportation Research Part B: Methodological 15: 105-111.

27. Wang, H, Kearney, J, Cremer, J, Willemsen, P (2005) Steering behaviors for autonomous vehicles in virtual environments. Proceedings of the IEEE virtual reality conference, Bonn, Germany, pp 155-162.

28. Cohn, A, Renz, J (2008) Qualitative spatial representation and reasoning. In: Handbook of knowledge representation. Elsevier, 3: 551-596.

29. El Hadouaj, S, Drogoul, A, Espié, S (2000) How to combine reactivity and anticipation : the case of conflicts resolution in a simulated road traffic. In: Moss, S, Davidsson, $\mathrm{P}$ (eds.) Multiagent-based simulation, second international workshop. Springer, Boston, USA, pp 82-96.

30. Saad, F, Schnetzler, B (1994) Un modèle conceptuel des comportement des conducteurs en conduite en file sur autoroute. In: Rapport de convention, technical report, Inrets/Drast.

31. Duchon, AP, Kaelbling, LP, Warren, WH (1998) Ecological robotics. Adaptive Behavior 6(3-4): 473-507.

32. Norman, D (1999) Affordances, conventions and design. Interactions 6(3): 38-43.
33. Raubal, M (2001) Ontology and epistemology for agent-based wayfinding simulation. International Journal of Geographical Information Science 15(7): 653-665.

34. Murphy, R (1999) Case studies of applying gibson's ecological approach to mobile robots. IEEE Transactions on Systems, Man, and Cybernetics, Part A: Systems and Humans 29(1): 105-111.

35. Stoytchev, A (2005) Behavior-grounded representation of tool affordances. Proceedings of IEEE international conference on robotics and automation (ICRA), pp 3071-3076.

36. Kapadia, M, Singh, S, Hewlett, W, Faloutsos, P (2009) Egocentric affordance fields in pedestrian steering. Proceedings of the 2009 symposium on interactive $3 D$ graphics and games. ACM, pp 215223.

37. Papasimeon, M, Pearce, A, Goss, S (2007) The human agent virtual environment. Proceedings of the 6th international joint conference on autonomous agents and multiagent systems. Honolulu, Hawaii, pp 1-8.

38. Afoutni, Z, Courdier, R, Guerrin, F (2012) Modélisation de l'action située basée sur les affordances et la stigmergie. In 20e journées francophones sur les systèmes multi-agents (JFSMA'12), pp 117-126.

39. Ksontini, F, Mandiau, R, Espié, S, Guessoum, Z (2012b) Représentation égo-centrée de l'environnement virtuel dans le cadre de la simulation de trafic routier. In 20e Journées Francophones sur les Systèmes Multi-Agents, JFSMA, pp 171-180.

40. Tornros, J (1998) Driving behavior in a real and a simulated tunnel - a validation study. Accident Analysis \& Prevention 30: 497-503.

41. Lewis-Evans, B, Charlton, SG (2006) Explicit and implicit processes in behavioural adaptation to road width. Accident Analysis \& Prevention 38(3): 610-617.

42. Ksontini, F, Guessoum, Z, Mandiau, R, Espié, S (2013). Using ego-centered affordances in multi-agent traffic simulation. In: Gini, ML, Shehory, O, Ito, T, Jonker, CM (eds) International conference on Autonomous Agents and Multi-Agent Systems, AAMAS '13, Saint Paul, MN, USA, May 6-10, 2013, IFAAMAS, pp 151-158.

43. Allen, J (1981) An interval based representation of temporal knowledge. Proceedings of the seventh international joint conference on artificial intelligence, pp 221-226.

44. Aupetit, S, Espié, S. (2012) Analyse ergonomique de l'activité de conduite moto lors de la pratique de l'inter-files en région parisienne. Activités 9(2) : 48-70.

45. Six, L (2014) Vers un modèle de comportements de véhicules lourds en utilisant une méthode incrémentale basée sur la vérification et l'hystérésis : le modèle ARCHIPL. Thèse de doctorat, Université Pierre et Marie Curie.

46. Ksontini, F, Espié, S, Guessoum, Z, Mandiau, R (2012a) Traffic behavioral simulation in urban and suburban-representation of the drivers' environment. Advances in intelligent and soft computing 155: 115-125.

47. Ducreux, BO (2008) Comparaison entre deux-roues à moteur et voitures : profil de conduite, consommation de carburant et émissions des gaz d'échappement en utilisation quotidienne. Données et références. Ademe. 
